\title{
Extragalactic globular clusters in the near-infrared ${ }^{\star}$
}

\section{IC 4051 and NGC 3311}

\author{
M. Hempel ${ }^{1,2}$, D. Geisler ${ }^{3}$, D. W. Hoard ${ }^{4}$, and W. E. Harris ${ }^{5}$ \\ ${ }^{1}$ European Southern Observatory, Karl-Schwarzschild-Str. 2, 85748 Garching, Germany \\ e-mail: mhempel@eso.org \\ 2 Michigan State University, Department of Physics and Astronomy, East Lansing, 48824 MI, USA \\ e-mail: hempel@pa.msu.edu \\ 3 Departamento de Física, Facultad de Ciencias Físicas y Matemáticas, Universidad de Concepción, Casilla 160-C, \\ Concepción, Chile \\ e-mail: doug@kukita.cfm.udec.cl \\ 4 Spitzer Science Center, California Institute of Technology, 220-6 Pasadena, CA 91125, USA \\ e-mail: hoard@ipac.caltech.edu \\ 5 Department of Physics and Astronomy, McMaster University, Hamilton, Ontario L8S 4M1, Canada \\ e-mail: harris@physics.mcmasters.ca
}

Received 18 August 2004 / Accepted 28 April 2005

\begin{abstract}
We present the results of combined optical and near-infrared photometry for the globular cluster systems of the giant ellipticals IC 4051 and NGC 3311 . We use the reduced age-metallicity degeneracy in $(V-I)$ vs. $(V-H)$ color-color diagrams to derive the cumulative age distribution within the red sub-population of globular clusters and to search for age sub-populations. The age distribution is then compared to the one determined for simulated globular cluster systems in order to set constraints on the relative age and size of these globular cluster sub-populations. In both galaxies we find a significant fraction of globular clusters with ages between 2-5 Gyr. We also investigate the metallicity distribution in both systems. Small number statistics prevent us from making any definite statements concerning NGC 3311, but we find that the derived metallicity distribution of the IC 4051 clusters strongly depends on the assumed age distribution. Based on our most likely result that finds a large number of young/intermediate age clusters ( 2 Gyr) within the selected globular cluster sample, we find metallicity peaks at $\sim-0.2$ for the old clusters and +0.8 for the young clusters. Only few very metal poor clusters are found. However, the metallicity distribution within the young/intermediate globular cluster population is significantly affected by our choice of the applied Single Stellar Population model. The mean metallicity of the second generation of globular clusters changes from the above mentioned and extremely high +0.8 dex to +0.2 dex. Note that the model dependency becomes less severe with an increasing age of the cluster population.
\end{abstract}

Key words. galaxies: individual: NGC 3311 - galaxies: individual: IC 4051 - galaxies: evolution - galaxies: stellar content

\section{Introduction}

Globular cluster systems (GCSs) are widely used to probe the formation and evolution of their host galaxies (e.g. Searle \& Zinn 1978; Zepf \& Ashman 1993; Ashman \& Zepf 1998; Kissler-Patig et al. 2002) although they represent only a small fraction of the galaxy's total luminosity. Hosting an almost perfect single stellar population (SSP), globular clusters allow the investigation of galaxies which, due to their large distances, cannot be resolved into single stars. The investigation of massive early-type galaxies, not found in the neighborhood of the Milky Way, in particular suffers from the large distance drawback. The diffuse light of a galaxy is a composite of all underlying stellar populations which can not be disentangled, and

* Appendix A is only available in electronic form at http://www.edpsciences.org populations of several ages and/or metallicities might indeed be hidden in the integrated light (e.g. Larsen et al. 2003). Detailed information on the age structure of the galaxy is therefore very difficult to obtain from the diffuse integrated light. Globular clusters, on the other hand, are luminous enough to be observed even at distances of $\sim 100 \mathrm{Mpc}$. Their stars share the same age and metallicity, and especially early-type galaxies can host quite numerous GCSs (Ashman \& Zepf 1998). The availability of SSP models (e.g. Bruzual 2000; Bruzual \& Charlot 2003; Vazdekis 1999; Vazdekis et al. 2003; Maraston 2005) for a wide range of ages and metallicities, applied to deep photometric or spectroscopic data of GCSs, allows us to set constraints on their age and chemical composition (e.g. Goudfrooij et al. 2001a; Puzia et al. 2002; 2004b; Hempel et al. 2003).

This basic tool allows extragalactic GC research to probe a variety of important questions. Since the discovery of the 
bimodal color distributions in globular cluster systems, their origin has been a source of ongoing debate, although there is general agreement that distinct globular cluster populations of different age and/or metallicity are the origin of this bimodality. When and how these populations were formed and what can be inferred for the evolution of the host galaxy are some of the most outstanding questions addressed today, with crucial implications for galaxy formation theories. Consequently, much effort has been put into the observation of GCSs in earlytype galaxies, which were classically assumed to have formed early on and to have evolved passively since then. From previous surveys, it is known that early-type galaxies are, on the one hand, well represented by the fundamental plane, indicating basic similarities, whereas their GCSs can show significant differences; for example with regards to metallicity (e.g. Geisler et al. 1996), color distribution (Gebhardt \& Kissler-Patig 1999), and specific frequency (Harris \& van den Bergh 1981; Harris 1991, 2001). Therefore, much caution has to be paid before claiming a universal formation scenario for early-type galaxies. In fact, it seems more and more likely that we have to abandon the idea of a single formation theory and find external parameters which might be decisive for the particular formation and evolution of a given galaxy. Factors involving the galaxy environment, such as its position within the host galaxy cluster/group or its isolation from other galaxies, is likely to be one of the most important external parameters. Galaxy interactions (e.g. mergers and accretion events) seem to be an important, if not the dominant, process, governing galaxy formation and evolution (see contributions in Wielen 1990). Consequently, the GCSs hosted by galaxies which were likely involved in such interactions need to be investigated. First results on the investigation of early-type galaxies in low-density environments were published by Kuntschner et al. (2002), who used integratedlight spectroscopy to derive ages and metallicities of $40 \mathrm{E}$ and S0 galaxies and conclude that early-type galaxies in a low density environment show a broad distribution of luminosityweighted ages and are on average younger than cluster ellipticals. Unfortunately, no early-type galaxies in a high galaxy density environment exist in the very local Universe. The determination of globular cluster ages by integrated-light spectroscopy is therefore quite challenging due to the faintness of the targets. The capability of high signal-to-noise spectroscopy with respect to age and metallicity determination has been demonstrated by various studies (e.g. Puzia et al. 2004a,b) and spectroscopy will doubtless be the method of choice when precise age estimates of a subsample of bright clusters are required. However, as long as the objective is to detect different broad age subpopulations in GCSs and to set coarse age limits on the GCS as a whole, then photometric studies (e.g. Kundu \& Whitmore 2001a,b; Gebhardt \& Kissler-Patig 1999) are the most efficient alternative.

Until recently, the major drawback for the use of photometric studies to investigate the age distribution in GCSs was the age-metallicity degeneracy (Worthey 1994). With the introduction of combined optical and near-infrared photometry (e.g. Minniti et al. 1996; Goudfrooij et al. 2001b; Kissler-Patig et al. 2002) it is now possible to at least partially overcome this obstacle and derive the relative ages of globular cluster sub-populations, as well as their metallicities. The extended wavelength range allows one to recognize intermediate age globular clusters (e.g. those formed in a recent merger) from the bulk of first generation objects, formed shortly after the Big Bang. In addition, colors like $V-H$ provide a sensitive metallicity index. The integrated IR colors for Galactic globular clusters of Cohen \& Matthews (1994) show that the $V-H$ index has three times the metallicity sensitivity of $V-I$, making it a good index for investigating the reality of any multiple metallicity populations.

In this study we investigate the GCSs of two important early-type galaxies, viz. NGC 3311, representing a giant cD galaxy at the center of a high density environment (the Hydra I galaxy cluster), and IC 4051, a giant E2 in the Coma galaxy cluster with no luminous neighbor, located several cluster core radii from the center. This will provide a range of environmental densities in order to begin to probe their effect on the GCS. The GCS of NGC 3311 has been investigated in the optical by many different groups (e.g. Grillmair et al. 1994; McLaughlin et al. 1995; Brodie et al. 2000; Secker et al. 1995). They found a populous GCS which appears to show the general color bimodality of such systems. Due to its central position in a high density environment, several wide field surveys have been carried out (e.g. Harris et al. 1983; Hilker 2003a,b) to investigate spatial features in the GCS. NGC 3311 is of special interest in globular cluster studies, since it is not only the third closest cD galaxy, following in distance M 87 in Virgo and NGC 1399 in Fornax, but also hosts one of the richest GCSs known (e.g. Harris et al. 1983; McLaughlin et al. 1995). The GCS of IC 4051 has only been attainable until now from HST imaging and was the subject of papers by Baum et al. (1997) and Woodworth \& Harris (2000). The latter work used $V, I$ photometry and found tentative evidence of bimodality, with metal-intermediate $([\mathrm{Fe} / \mathrm{H}] \sim-0.7)$ and metal-rich ( $\sim$ solar) peaks and a near-complete lack of metal-poor clusters. The lack of metal-poor clusters and the very high specific frequency $\left(S_{N}=11 \pm 2\right)$ that they found makes the GCS of IC 4051 very unique and worthy of further study. General information concerning these 2 galaxies can be found in Table 1 .

In this paper, we combine optical and near-infrared photometry to derive the cumulative age distribution (e.g. Hempel $\&$ Kissler-Patig 2004) which we will compare to simulated GCSs of known age structure (size and age of sub-populations). In this particular case the model isochrones by Bruzual \& Charlot (2003) have been applied and the results are discussed in great detail. Nevertheless, an additional section will be spent to discuss the dependency of the results on the choice of SSP models. To do so we will repeat the analysis and apply the latest version of the SSP models by Maraston (2005). These results enable us to set constraints on the age structure in both systems and to infer clues to the evolutionary history of their host galaxies (Hempel \& Kissler-Patig 2004). The paper is organized as follows. In Sect. 2 we describe the observations as well as the data reduction. The derivation of the cumulative age distribution within the observed samples is described briefly in Sect. 3 as well as the results of this analysis. For a detailed description we refer to Hempel et al. (2003) and Hempel $\&$ Kissler-Patig (2004). In Sect. 4 we derive and discuss the 
Table 1. General information about the host galaxy.

\begin{tabular}{lrrl}
\hline \hline Property & NGC 3311 & IC 4051 & Reference \\
\hline RA(J2000) & $10 \mathrm{~h} \mathrm{36m} \mathrm{43s}$ & $13 \mathrm{~h} 00 \mathrm{~m} \mathrm{55s}$ & deVaucouleur et al. (1991) \\
Dec(J2000) & $-27^{\circ} 31^{\prime} 42^{\prime \prime}$ & $+28^{\circ} 00^{\prime} 27^{\prime \prime}$ & deVaucouleur et al. (1991) \\
$B_{\mathrm{T}, 0}$ & 12.65 & 14.17 & deVaucouleur et al. (1991) \\
$E_{B-V}$ & 0.079 & 0.011 & Schlegel et al. (1998) \\
$A_{V}$ & 0.263 & 0.035 & Schlegel et al. (1998) \\
$A_{I}$ & 0.154 & 0.02 & Schlegel et al. (1998) \\
$A_{H}$ & 0.046 & 0.006 & Schlegel et al. (1998) \\
$(B-V)_{\mathrm{eff}}$ & 1.04 & 1.01 & deVaucouleur et al. (1991) \\
$(m-M)_{V}$ & $33.70 \pm 0.08$ & $34.82 \pm 0.13$ & Jensen et al. (2001) \\
$M_{V}$ & $-22.07 \pm 0.20$ & $-21.62 \pm 0.35$ & deVaucouleur et al. (1991) \\
\hline
\end{tabular}

Table 2. Exposure times for NGC 3311 and IC 4051.

\begin{tabular}{lrr}
\hline \hline Filter (Instrument) & NGC 3311 & IC 4051 \\
\hline$F 814 W($ WFPC2) & $3800 \mathrm{~s}$ & $5200 \mathrm{~s}$ \\
$F 555 W($ WFPC2) & $3700 \mathrm{~s}$ & - \\
$F 606 W($ WFPC2) & - & $20500 \mathrm{~s}$ \\
$F 160 W($ NICMOS2) & $2560 \mathrm{~s}$ & $2560 \mathrm{~s}$ \\
\hline
\end{tabular}

metallicity distribution. We summarize our results in Sect. 5 . We will dedicate Appendix A to the discussion of how the results are influenced by our choice of a Single Stellar Population model.

\section{Observations and data reduction}

The optical and the near-infrared data for both galaxies were reduced by different groups, using different software. Therefore we will give a brief description for each data set.

\subsection{Optical data: HST/WFPC2}

Both targets were observed with HST + WFPC2 in two optical pass bands, NGC 3311 in $F 814 W$ and $F 555 W$, and IC 4051 in $F 814 W$ and $F 606 W$, respectively. NGC 3311 observations are part of a large survey on extragalactic GCSs, carried out under program number GO.6554. The observations of IC 4051 were carried out under program GO.6283, dedicated to stellar populations in elliptical galaxies. The total exposure times in the various pass bands are given in Table 2 for both targets.

For IC 4051 we took the photometry directly from Woodworth \& Harris (2000). The reduction of the optical data for NGC 3311 is described in the following.

The photometry on NGC 3311 clusters was done using the DAOPHOT package for aperture- and alternatively for $p s f$ photometry as well as using SExtractor (Bertin \& Arnout 1996) aperture photometry. In Fig. 1 we show the comparison between $(V-I)$, calculated from instrumental $V$ and $I$ band magnitudes, using DAOPHOT $p s f$-photometry and SExtractor aperture-photometry for all objects detected on the PC1 chip,

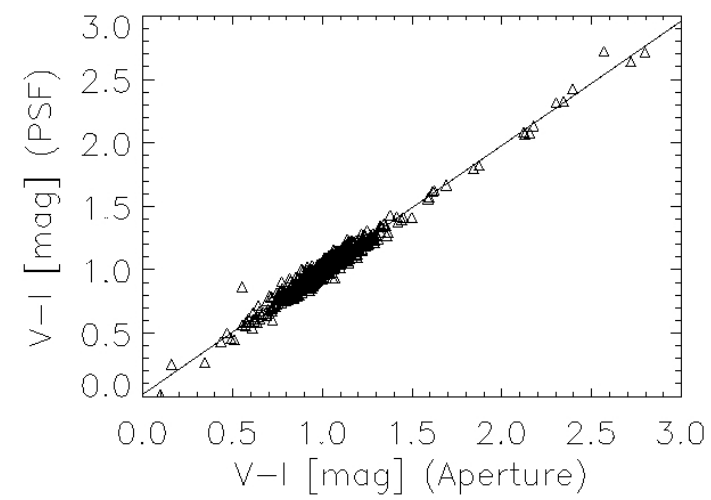

Fig. 1. Comparison between $(V-I)$ colors based on aperture and $p s f$ photometry. The solid line represents the least-squares fit to $(V-I)$ based on aperture photometry. The objects in this plot contain the $(V-I)$ colors for the PC1 chip as well as for all three wide field chips. As mentioned in Sect. 2 the latter could not be included in our age analysis, due to the smaller spatial coverage of the near-infrared observations.

for which later on also $H$-band data were obtained. The extremely red objects $((V-I)>1.6)$ have been rejected for being objects either in the vicinity of the central dust shell found in the galaxy core or close to the edge of the PC chip. Including an error selection, i.e. $\delta(V-I)<0.15 \mathrm{mag}$, the results of a least square fit between the $(V-I)$ values are:

$(V-I)_{\mathrm{aperture}}=0.983(V-I)_{\mathrm{psf}}+0.015$.

There is no difference between the results, the color offset being smaller than the photometric error, except for very red objects $((V-I)>2.0 \mathrm{mag})$. The NGC 3311 results presented in this Paper are based on SExtractor photometry.

The instrumental magnitudes were transformed to the Johnson $V$ and Cousin $I$ magnitudes according to the procedure given by Holtzman et al. (1995) and reddening corrected using the values given in Table 1 . The aperture corrections for both filter bands were determined as the mean difference ( 21 objects) between a 6 pixel instrumental magnitude and the 0 .'5 aperture used by Holtzman et al. (1995). The calculated corrections, $0.229 \mathrm{mag}$ and $0.265 \mathrm{mag}$ for the $V$ and $I$ band, 
respectively, were than applied to all of the $V$ - and $I$-band detected objects. The small aperture was necessary because of crowding.

The near-infrared data used in this paper were derived from the instrumental magnitudes obtained in the non-standard filter systems of NICMOS, using the information of the mean metallicity based on optical colors. The relation between $(V-I)$ and the metallicity is rather well calibrated, using the globular clusters of the Milky Way or M31, respectively. However, considering the fact that the correction between $F 160 \mathrm{~W}$ and the Cousin $H$-band for a mean metallicity of $\langle\mathrm{Fe} / \mathrm{H}\rangle \sim-0.3$ (IC 4051) and $\langle\mathrm{Fe} / \mathrm{H}\rangle \sim-0.75$ (NGC 3311), as described in Sects. 2.2 .1 and 2.2 .2 differ only insignificantly $(0.115 \mathrm{mag}$ in IC 4051 compared to $0.110 \mathrm{mag}$ in NGC 3311) leads us to the conclusion that the a priori assumption of the metallicity does not alter the final outcome of our analysis. Nevertheless, since additional near-infrared data are desirable for an improved GC sample in both galaxies, observations using broad band filters with a response function closer to the JohnsonCousin system are clearly preferable, or alternatively observations in multiple bands, allowing a proper color correction.

\subsection{Near-Infrared Data: HST/NICMOS2}

Both galaxies were observed with NICMOS Camera 2 (fov: 19 !' $2 \times 19$ !' $2,0.075 \mathrm{pix}^{-1}$ ) through the $F 160 \mathrm{~W}$ filter (approximately $H$ band) in a slightly overlapping ( $\sim 10-20$ pixels) fourpoint mosaic pattern, centered on the galaxy. The combined mosaic image covers approximately the same area and galaxy field as the WFPC2 PC1 chip. Each of the four quadrants is a combination of 5 sub-images. The exposure time for each subimage is $512 \mathrm{~s}$, the total exposure time sums up to $2560 \mathrm{~s}$ (see Table 2). As for the optical filter bands, we give the details for data reduction separately for each target.

\subsubsection{IC 4051}

In IC 4051 the SExtractor routine (Bertin \& Arnout 1996) was used to identify all targets in the four quadrant images of the galaxy-subtracted NICMOS2 image. This resulted in the detection of a total of 642 targets. The aperture photometry was then carried out using the non-standard IRAF task CCDCAP (Mighell \& Rich 1995), which is optimized for aperture photometry of undersampled images. Hereafter, the photometry procedure recommended in the NICMOS Data Handbook ${ }^{1}$ (Dickinson et al. 2002) was applied. The instrumental magnitudes were measured with an aperture of 0.'5 (radius, corresponding to 6.6 pixel) and corrected to "infinite" aperture radius by adding -0.1517 mag, calibrated by adding 23.566 mag (which is the magnitude of a star with count rate of 1 electron per second in the NICMOS $F 160 W$ filter), and corrected for the total quadrant image exposure time. Finally, we performed a transformation from the NICMOS F160W filter magnitudes to standard $H$ magnitudes (Stephens et al. 2000). Based on the mean metallicity of IC 4051 globular clusters of $\langle\mathrm{Fe} / \mathrm{H}\rangle \sim-0.1$ (Woodworth \& Harris 2000), combined with observations of

\footnotetext{
1 See http:www.stsci.edu/hst/nicmos/
}

Galactic and M 31 globular clusters (Barmby et al. 2000), leads to the following transformation:

$H=F 160 W-0.115( \pm 0.048) \mathrm{mag}$.

After all calibration corrections were applied to the instrumental magnitudes, a number of targets were rejected: all targets within 12 pixels of any edge of a quadrant image, all targets for which CCDCAP failed to measure a magnitude, all targets with SExtractor star/galaxy indices less than 0.5 (indicating they are likely galaxies), targets located near the the center of the subtracted galaxy (within $\sim 2$ '. 0 , where photometric errors are very large due to the background galaxy light), and for identical targets located within the overlapping regions of two quadrant images, the one with the larger uncertainty. A total of 348 objects remained. After transforming the WFPC2 pixel coordinates to the NICMOS coordinates, and allowing an offset of up to 2 pixels in both $x$ and $y$ coordinates, the optical and near-infrared data were matched. The final sample now includes 256 objects.

\subsubsection{NGC 3311}

As in the optical filter bands the source detection on the NICMOS images was done using DAOPHOT/PHOT as well as SExtractor. To be as consistent in the data reduction as possible, we will stay with the SExtractor results. The transformation of the $F 160 \mathrm{~W}$ data to the Johnson $H$ band followed the procedure described in Sect. 2.2.1 and results in the following correction:

$H=F 160 W-0.110( \pm 0.024) \mathrm{mag}$.

The differences in the transformation between $F 160 \mathrm{~W}$ and $H$-band magnitudes between NGC 3311 and IC 4051 originates in the difference of the mean metallicity, derived from the $(V-I)$ distribution, where we have adopted a mean metallicity of $\langle\mathrm{Fe} / \mathrm{H}\rangle \sim-0.75$ (Brodie et al. 2000). Besides the F160W- $H$ band transformation, the instrumental magnitudes were aperture corrected, the photometric zero point for NICMOS2 applied and finally corrected for galactic reddening (see Table 1). After combining optical and near-infrared data the globular cluster sample includes 148 objects.

In this series of papers we concentrate on the age structure in globular cluster systems, which we derive from color-color diagrams. The basic tool in this approach (see Sect. 3.2 and Hempel \& Kissler-Patig 2004) is the color distribution of the observed globular clusters with respect to SSP model isochrones. Therefore we have to consider the contamination of the globular cluster samples. Considering the color range, the contaminants are most likely background galaxies. Although we apply selection criteria, e.g. shape parameters and color limits, we are aware that contamination is still an issue. Nevertheless, the final globular cluster samples, combining optical and near-infrared data, contain only objects in the very central region of the GCS, where we find the highest globular cluster density. In combination with the sample size ( $\approx 100$ objects), as discussed in Sect. 3.2, we estimate that unresolved background galaxies make only a minor contribution to our sample and should have a minimal effect on the age distribution. 


\section{Results}

\subsection{Color-color distributions}

Our basic tools to derive ages and metallicities of stellar populations from photometric data are color-color diagrams together with various SSP models; for example by Bruzual \& Charlot (2000, 2003), Vazdekis (1999), Maraston et al. (2001a) or their most recent release (Maraston 2005). We will use the Bruzual \& Charlot models (Bruzual \& Charlot 2003) throughout the main body of the paper whereas Appendix A is based on the Maraston models (Maraston 2005) which indicates that only minor differences occur between models for ages $>1 \mathrm{Gyr}$ and that our main goal of detecting different aged subgroups is not compromised. In our further analysis we select only clusters which satisfy an error limit of $\delta_{(V-I)}$ and $\delta_{(V-H)}<0.15 \mathrm{mag}$. The error limit was derived as a combination of the photometric error in two filter bands with $\delta \leq 0.1$ mag each, corresponding to a $S / N=10$ for detected objects. The error selected globular cluster samples for NGC 3311 and IC 4051 contain 105 and 149 objects, respectively. In our further analysis of the age structure additional color cuts will be applied to minimize contamination with unresolved background objects and to select objects within a restricted color range that allows the best age resolution (see Sect. 3.2). With respect to previous studies, e.g. by Hilker (NGC 3311, Hilker 2002, 2003a) and Woodworth \& Harris (IC 4051, Woodworth \& Harris 2000) we note the much smaller size of our sample, which is mostly due to the included $H$-band data, and the small field of view of the NICMOS2 instrument. As we will discuss later (see Sects. 3.2 and 5), the limited spatial distribution of the globular cluster sample becomes of importance for our interpretation of the derived relative sizes of the age sub-populations.

\subsubsection{IC 4051}

In Figs. 2 and 3 we show the color-magnitude diagram (CMD) and the $(V-I)$ vs. $(V-H)$ color-color diagram for IC 4051. Clearly, the data below the $50 \%$ completness limit of the $H$ photometry are unreliable and will be excluded from further analysis, as will all objects with color errors above our error cut. In Woodworth \& Harris (2000) it was tentatively suggested that the $(V-I)$ distribution of the IC 4051 globular clusters is bimodal and, therefore, we attempted to fit a double Gaussian function to the $(V-H)$-color distribution, which is three times more sensitive to metallicity than $(V-I)$. If the complete sample set is included in this analysis, then comparably good fits are obtained for variable amplitudes for both sub-populations or (forced) equal amplitude, equal width settings. In the latter case the Gaussian centers are at $(V-H)=2.70$ and 3.18 . The mean of these two Gaussian centers is 2.94 , which is approximately equal to both the mean of all colors and the center of the single Gaussian fit. Thus, this fit essentially devolves to merely reproducing the single Gaussian case. However, since uni- and bimodal fits give similarly good results (see Fig. 4), we will stay with the assumption of a unimodal $(V-H)$ color distribution and a corresponding mean color of $\langle V-H\rangle=2.92$. We note that the situation changes drastically if we apply the

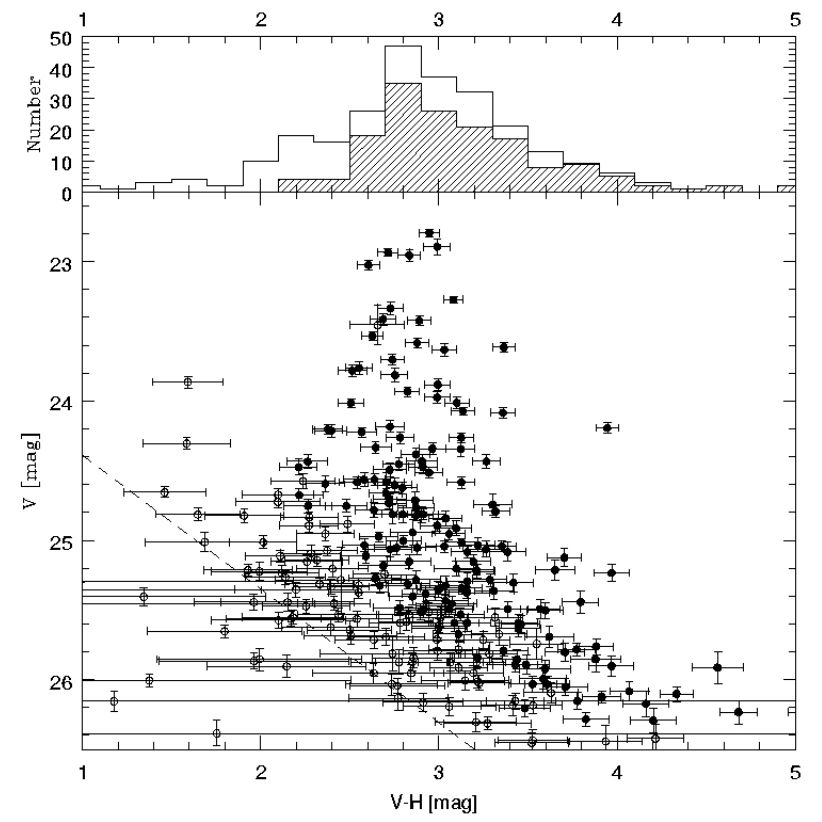

Fig. 2. $V$ vs. $(V-H)$ color-magnitude diagram for IC 4051. The top sub-panel shows the color distribution of all (open histogram) and error-selected (hashed histogram) objects. The lower sub-panel shows the color-magnitude diagram. Herein the filled symbols mark the selected clusters, while the open circles mark rejected objects. The dashed line marks the $50 \%$ completeness limit for the $H$-band.

selection by error limit. As we can see in Fig. 2, the blue population $(V-H<2.4)$ is significantly diminished by the error limit, which makes it difficult to draw strong conclusions from the color distribution.

With the discovery of bimodal color distributions in globular cluster systems (e.g. Whitmore \& Schweizer 1995; Geisler et al. 1996; Kundu \& Whitmore 2001a; Gebhardt \& Kissler-Patig 1999) the interest in the GCSs of early-type galaxies, which were supposedly old and uniform, grew immensely. The spatial distribution of GCs adds an extra clue to their origin. For example, in the Galaxy, metal rich GCs are found to be more confined to the Galactic disk out to distances up to $\sim 10 \mathrm{kpc}$ from the Galactic center (Searle \& Zinn 1978), and metal-poor clusters are found at even larger distances but spherically distributed in the Galactic halo (Ashman \& Zepf 1998). Although the near-infrared data for IC 4051 cover only the innermost region of IC 4051 (maximum distance $\sim 9 \mathrm{kpc}$ ) it is still worth investigating the radial distribution of the globular clusters mean colors. Figure 5 shows the distribution of globular cluster colors as a function of galactocentric angular distance $R$; we have listed the mean colors in $2 . ' 5$ wide bins in Table 3. We note that there is no obvious trend in the mean colors. $V-H$ is primarily sensitive to metallicity, so this indicates the lack of any strong metallicity gradient in the IC 4051 GCS, for radial distances $R \leq 22$ '. 5 .

Comparing the combined optical and near-infrared colors with SSP model isochrones (see Fig. 3) reveals that only a fraction of the red cluster population is consistent with an old $(>10 \mathrm{Gyr}$ ) population. We find a large fraction of objects whose $(V-I)$ color at a given $(V-H)$ is too blue to fit into an "old" 


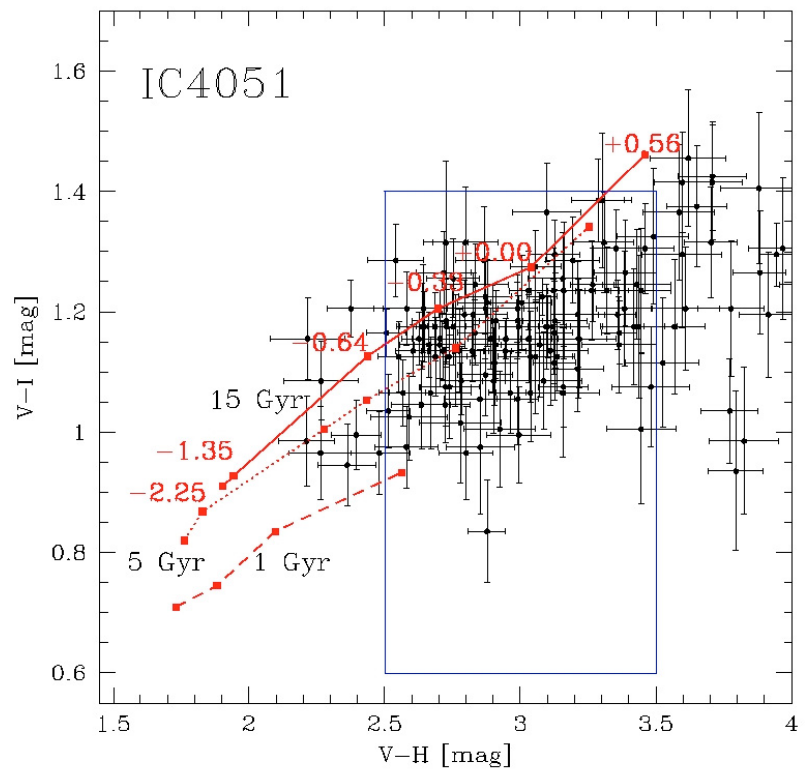

Fig. 3. $(V-I)$ vs. $(V-H)$ color-color diagram for IC 4051. All data are corrected for foreground reddening (see Table 1). The $15 \mathrm{Gyr}, 5 \mathrm{Gyr}$ and 1 Gyr isochrones are based on the Bruzual \& Charlot SSP models (Bruzual \& Charlot 2003). The solid squares mark the metallicity in $[\mathrm{Fe} / \mathrm{H}]$, increasing with $(V-H)$ from -2.25 up to +0.56 . The box marks the color range selected for the determination of the cumulative age distribution.

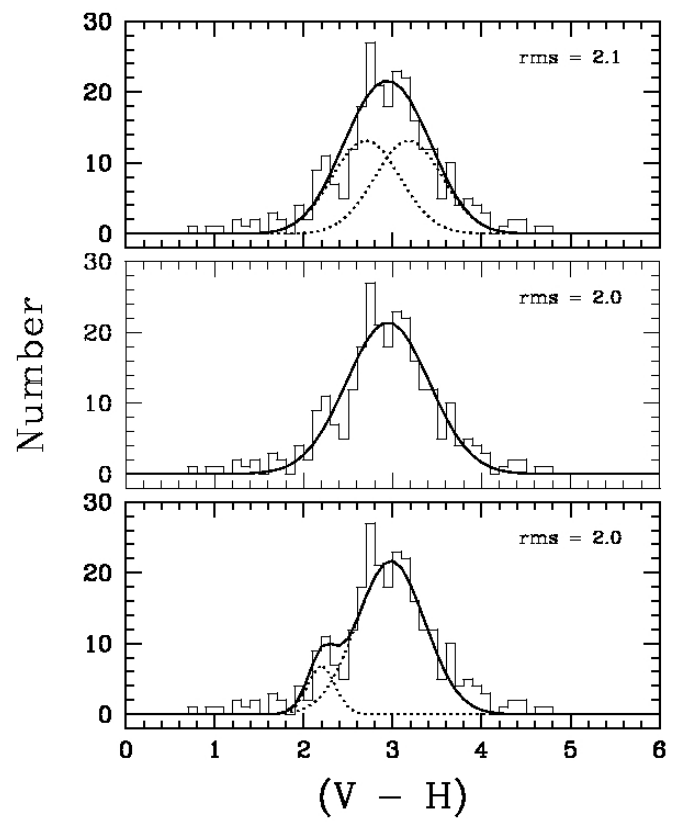

Fig. 4. $(V-H)$ color distribution in the complete IC 4051 sample showing a single Gaussian fit (middle) and two different double Gaussian fits (top: equal amplitude/equal width, bottom: variable amplitude/variable width). The dotted lines mark the contribution of each individual component, solid lines are their sum.

globular cluster scenario. Their color is much better matched by an intermediate age population (i.e. with an age between $\sim 1$ and $7 \mathrm{Gyr}$ ). Such a large intermediate/young population of globular clusters seems suspicious, since the integrated galaxy light does not show any hint of a recent major star formation

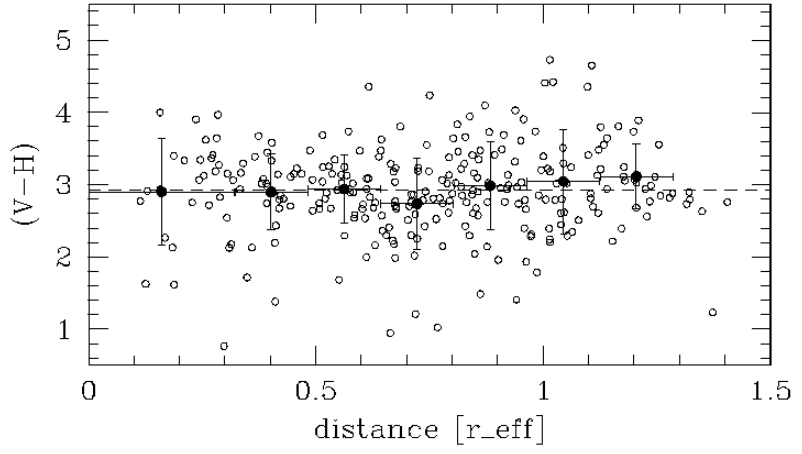

Fig. 5. Distribution of globular cluster colors for IC 4051 as a function of radius from the center of the galaxy (open circles). The dashed line marks the mean value of the entire data set, $\langle V-H\rangle=2.922 \mathrm{mag}$. The solid circles show the mean colors in increasing radius bins, as listed in Table 3.

Table 3. Mean color versus radius for IC 4051.

\begin{tabular}{rccc}
\hline \hline Radius (") & $N$ & $\langle V-H\rangle$ & $\sigma_{V-H}$ \\
\hline$<5.0$ & 30 & 2.901 & 0.745 \\
$5.0-7.5$ & 28 & 2.897 & 0.530 \\
$7.5-10.0$ & 41 & 2.938 & 0.478 \\
$10.0-12.5$ & 51 & 2.737 & 0.635 \\
$12.5-15.0$ & 51 & 2.984 & 0.612 \\
$15.0-17.5$ & 38 & 3.044 & 0.724 \\
$17.5-20.0$ & 25 & 3.114 & 0.451 \\
\hline
\end{tabular}

event and it is a relatively isolated galaxy. With respect to the integrated light argument we refer to the work by Puzia et al. (2002) on NGC 4365 who found a similar scenario where the combined $(V-I)$ and $(V-K)$ color of the host galaxy is consistent with a metal-rich and relatively old stellar population although the globular cluster system indicates a significant fraction of intermediate age globular clusters as well as old ones (Puzia et al. 2002; Hempel \& Kissler-Patig 2004). The apparent contradiction between the two results breaks down if we compare the observed fraction of globular clusters with the stellar content of the galaxy. The derived ratios between age subpopulations in both galaxies refers to only a small sub-sample of the globular cluster system and is therefore subject to various bias effects, as will be discussed later. In this respect we also note the most recently published results on NGC 4365 by Brodie et al. (2005). In this paper spectral data of GCs are used to derive their ages, which seem to contradict previously obtained age estimates, both spectroscopic and photometric, and claim an average age of $11 \mathrm{Gyr}$ for the complete GC sample, with no evidence for intermediate age clusters. At this point we have to take into account that the different samples have only few globular clusters in common. However, the authors discuss the possibility of spatial distribution effects, which is indeed an important issue when drawing conlusions on ages of selected samples. Clearly more data are required to explain the different results. 
At this point we want to emphasize that only within the red sub-population is it possible to distinguish the age of globular clusters whereas the blue population should represent only the first generation, but deriving even crude age estimates for such blue clusters is very difficult. This supposedly old fraction is NOT included in the derived size of the different age populations. In Sect. 1 we mentioned a gas rich merger as a possible scenario to form a second generation of globular clusters, which requires a certain minimum galaxy density. IC 4051 is found somewhat outside the core of the Coma galaxy cluster, surrounded by numerous other galaxies, but not in the center of the gravitational potential of the galaxy cluster. However, additional evidence for a less passive evolution of this galaxy is the existence of a co-rotating galaxy core, which rotates at a different velocity from the main galaxy body. It is not known yet whether it is dynamically decoupled or not (Mehlert et al. 1998). Such peculiarities are quite common in early-type galaxies (e.g. Davies et al. 2001; de Zeeuw et al. 2002) and we do not intend to link the existence of such a co-rotating core directly with a second star formation event. However, if the dynamical structure of an early-type galaxy sets it apart from the formerly- uniform and simple structured picture, then these will be prime targets to look for multimodal age distributions. In this respect we want to emphasize that our classification of IC 4051 as an early-type galaxy in a less dense environment is rather relative, since the Coma galaxy cluster represents a high density environment (projected central galaxy density of $\approx 39$ galaxies/ $\mathrm{Mpc}^{2}$, Kent \& Gunn 1982) compared to the Hydra I galaxy cluster (17 galaxies/ $\mathrm{Mpc}^{2}$, Richter 1989). Nevertheless, due to its distance of 15.5 ( 460 kpc, see Jorgensen \& Franx 1994) from the cluster center we do not consider IC 4051 as a galaxy in a high density environment anymore.

\subsubsection{NGC 3311}

The $V$ vs. $(V-H)$ color-magnitude diagram and $(V-I)$ vs. $(V-H)$ color-color diagram for NGC 3311 are presented in Figs. 6 and 7. The histogram in the upper part of the CMD shows the color distribution of the complete sample as an open diagram, whereas the error selected sample is shown by the hatched histogram.

The color-color distribution in Fig. 7 shows that in addition to many old GCs, a large fraction of red globular clusters $(V-H>2.5)$ have a $(V-I)$ color which is too blue to be consistent with an old cluster population. A direct comparison to the SSP isochrones assigns those objects an age $\$ 5 \mathrm{Gyr}$. The possibility of a young/intermediate population of globular clusters formed in a merger was already discussed by Hilker (2002). Our near-infrared observations do not favor the second possibility, viz. an old and metal poor population. Interestingly, NGC 3311 contains a prominent dust lane in its center (e.g. Västerberg et al. 1991; Grillmair et al. 1994), which adds further evidence to support a recent merging or accretion event. The dust content in the core of NGC 3311 was estimated as $10^{3.5} M_{\odot}$ (Ferrari et al. 1999) based on $V$ and $R$-band photometry. Grillmair et al. (1994) derived a lower limit

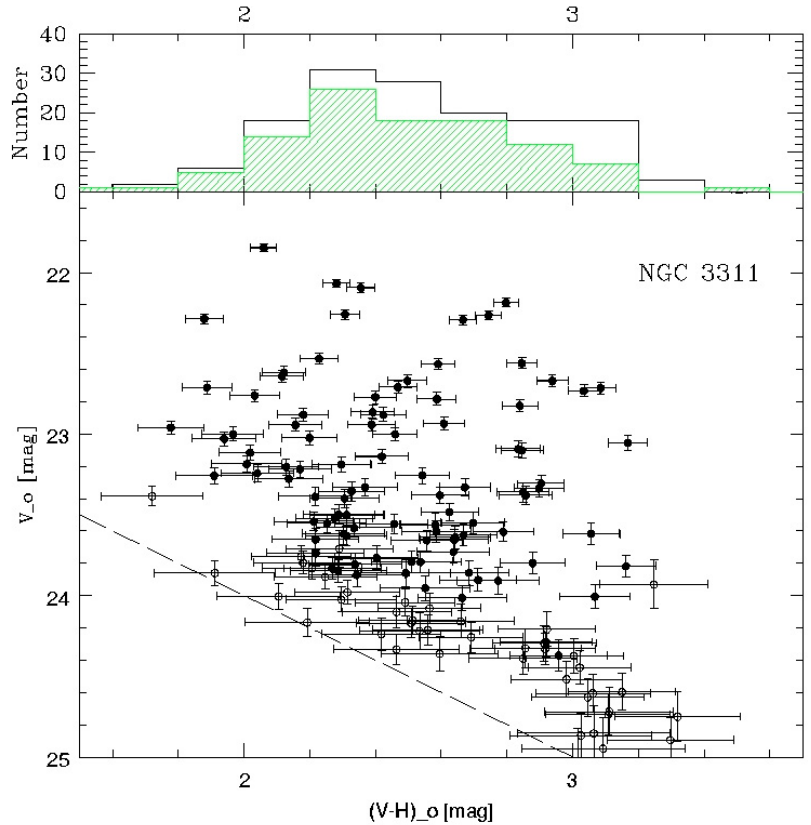

Fig. 6. $V$ vs. $(V-H)$ color-magnitude diagram for NGC 3311. The top sub-panel shows the color distribution of all (open histogram) and error-selected (hashed histogram) objects. The lower sub-panel shows the color-magnitude diagram. Herein the filled symbols mark the selected clusters, while the open circles mark rejected objects (by error cut). The dashed line marks the 50\% completeness limit for the $\mathrm{H}$-band, derived from the detection rate in artificial data sets using the Addstar routine in the IRAF package.

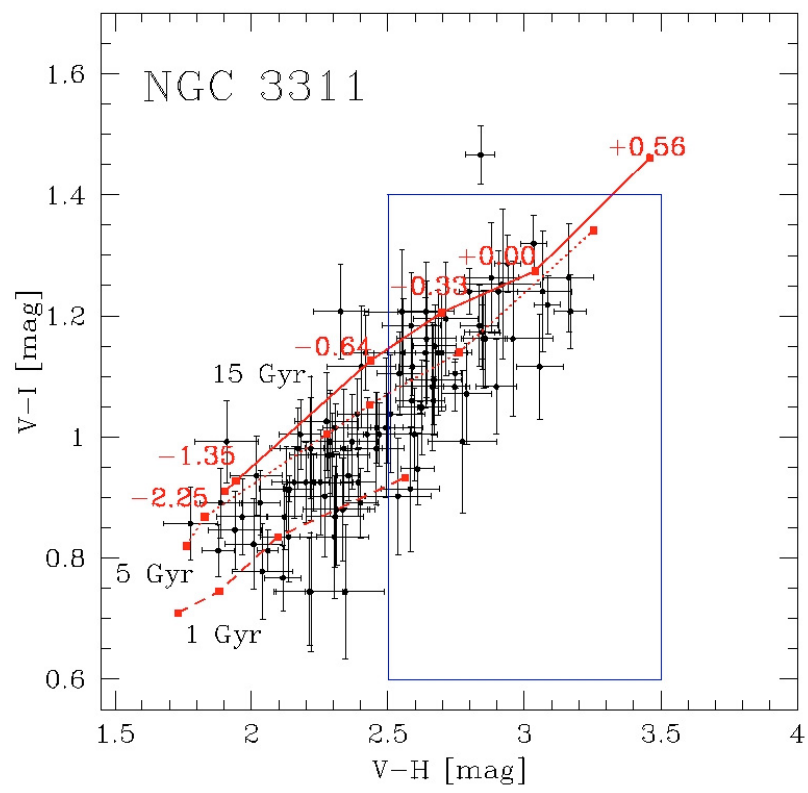

Fig. 7. $(V-I)$ vs. $(V-H)$ color-color diagram for NGC 3311 . The data obey the selection by photometric error cut $(<0.15 \mathrm{mag})$ and are corrected for foreground reddening (see Table 1). As examples the $15 \mathrm{Gyr}, 5 \mathrm{Gyr}$, and $1 \mathrm{Gyr}$ isochrones, corresponding to the Bruzual \& Charlot model isochrones (Bruzual \& Charlot 2003) are marked. The metallicity, rising from $[\mathrm{Fe} / \mathrm{H}]=-2.25$ to $[\mathrm{Fe} / \mathrm{H}]=+0.56$, is marked by solid squares. The color range selected for the determination of the cumulative age distribution is marked by the box. Compared to IC 4051 the $(V-H)$ color range is slightly shorter to fit the observed data. 
of $4.6 \times 10^{4} M_{\odot}$ for the total dust mass. Assuming a similar gas-to-dust ratio as found in the Galaxy, $m_{\text {gas }} / m_{\text {dust }} \sim 100$ (Jura 1986; McNamara et al. 1990), the total gas and dust mass will reach at least $4.6 \times 10^{6} M_{\odot}$. The globular cluster sample included in our analysis, however, refers only to the innermost region in NGC 3311. Additional near-infrared observations covering larger galactocentric radius will be necessary before any conclusion can be inferred from comparing our small central sample to the entire blue globular cluster population found by Hilker (2002), using optical photometry alone. As previous studies on NGC 4365 (Puzia et al. 2002) have shown, results based on optical photometry alone do not always indicate the existence of age sub-populations. The distribution of $(V-I)$ colors shows only the common bi-modal distribution, mostly caused by the metallicity. Only the combination with near-infrared colors reveals the more complex age structure of the globular cluster system.

\subsection{Cumulative age distribution}

Our technique to derive cumulative age distributions, as described in Hempel et al. (2003) and Hempel \& Kissler-Patig (2004), is to help lift the age-metallicity degeneracy (Worthey 1994) via the combination of optical and near-infrared broad band colors. Hereby the higher sensitivity of infrared colors to metallicity as compared to age is utilized. An example of the comparison of the age and metallicity dependency of $(V-I)$ and $(V-K)(\sim(V-H))$ can be found in Puzia et al. (2002) and references therein. The cumulative age distribution is then defined as the percentage of objects older than T Gyr, and is derived by comparing their $(V-I)$ color with the SSP model prediction for a population that is T Gyr old. The lowest age bin is assigned to $0 \mathrm{Gyr}$ and includes all GCs within the selected $(V-H)$ color range. With respect to a possible formation scenario of the GCSs and their host galaxies, we want to go one step further and set constraints on the age and the relative size of the cluster sub-populations. The method is based on the comparison between observed and simulated GCSs (Hempel et al. 2003; Hempel \& Kissler-Patig 2004) via a $\chi^{2}$-test. To simulate the color distribution in GCSs and to derive the age distribution in simulated and observed systems, the SSP model isochrones of Bruzual \& Charlot (2003) were again used. Examples for the simulated color-color diagrams and the resulting cumulative age distributions are shown in Fig. 8. Instead of a stepfunction-like age distribution we find a much more gradual slope in the age distribution, which is mostly due to the fact that the $(V-I)$ colors in our simulations are not strictly independent from metallicity effects and, therefore, show a certain dependency on $(V-H)$. The final consequence is that even with a much improved age resolution of combined optical and nearinfrared colors, the determination of accurate absolute ages for single globular clusters is still out of reach. Our intention is to detect age sub-populations, to set constraints on their age and to find systematic effects of external parameters on the age structure. In nearby galaxies more precise age determinations can be derived from spectroscopic spot checks. For our simulations we assume a composition of two age sub-populations
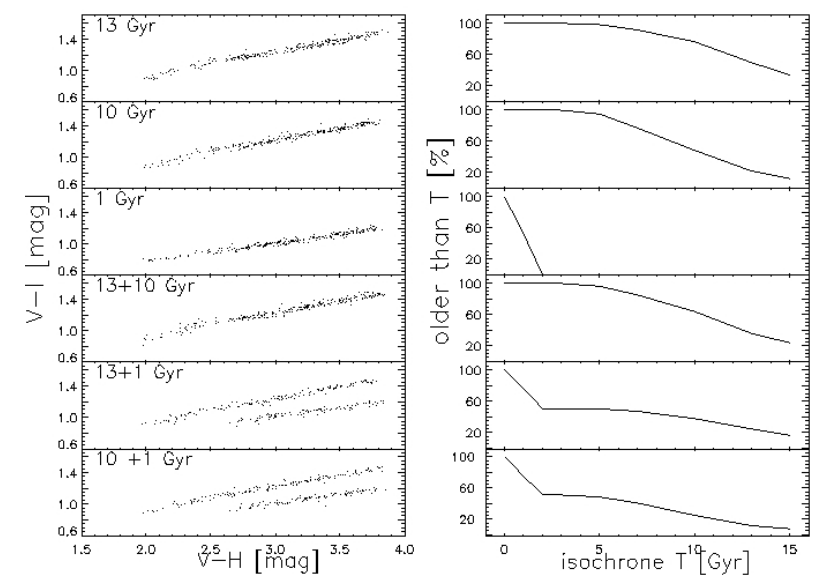

Fig. 8. Simulated color-color diagrams (left) and the resulting cumulative age distribution (right) for various age combinations. The two populations are equally mixed between the old and intermediate age populations. The ideal colors, following the Bruzual \& Charlot (2003) SSP models, were smeared randomly with an up to $3 \sigma$ photometric error.
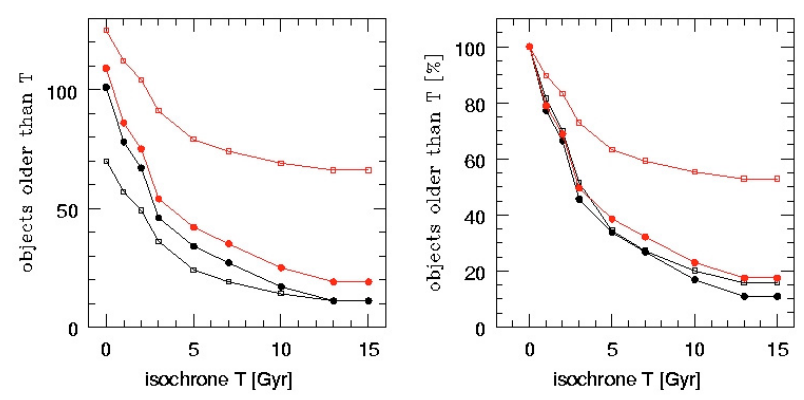

Fig. 9. Cumulative age distribution (dark line) in NGC 3311 (open squares) and IC 4051 (solid circles) derived following the procedure given in Sect. 3.2. The left panel shows the absolute numbers of globular clusters older than a given age. In the right panel the distribution was normalised with respect to the total number of objects found. If we assume the blue population of globular clusters $((V-H)<2.5)$ to consist of only $13 \mathrm{Gyr}$ old objects the cumulative age distribution would change as shown by the grey patterns.

with ages ranging from 1, 1.5, 2, 3, 5, 7, 10 and 13 Gyr. Hereby we vary the amount of young/intermediate age globular clusters between $100 \%$ and $0 \%$. The $\chi^{2}$-test finds the best fitting model with a given age and size ratio.

As shown in Sects. 3.1.2 and 3.1.1 the color-color diagrams give us a first indication for the existence of multiple age sub-populations in the GCSs of both NGC 3311 and IC 4051. If we compare the cumulative age distributions (Fig. 9) with the simulation of a purely old system (see Fig. 8, upper, right panel) the differences are obvious.

From Fig. 9, the age distribution in both GC samples assigns $\sim 65 \%$ of the globular clusters to an age younger than 5 Gyr. Recall that this refers only to GCs with $V-H>2.5$. Although the combination of optical and near-infrared colors helps lift the age-metallicity degeneracy, $(V-I)$ is still mildly affected by the metallicity. Combined with the scatter in both colors, induced by the photometric errors, and the relatively small sample size (IC 4051: 101 GCs, NGC 3311: 70 GCs), 
this results in a significant uncertainty with respect to the size estimate of the sub-populations. To overcome this obstacle we will not use the cumulative age distribution as a direct measure for the age structure in the GCSs, but rather compare the observations with the simulated systems via a $\chi^{2}$-test. In this test we determine the difference between the cumulative age distribution in observed and simulated systems. Nevertheless, we still have to accept a certain degeneracy between age and size of sub-populations, which results in a finite accuracy with respect to the best fitting model. Since the major goal of this project is to detect age sub-populations and set some constraints on their age, it is of no importance here (and impossible for us to detect) whether the second generation of globular clusters is 2 or $3 \mathrm{Gyr}$ old and contains 30 or $40 \%$ of the globular cluster sample. This uncertainty becomes even more important for NGC 3311, where a large fraction of the observed GCs are excluded from the age analysis by the color cut. In the comparison of our results with previously obtained age estimates we will therefore concentrate on IC 4051 (e.g. Mehlert et al. 1998; Mehlert et al. 2003). Based on spectroscopic data (Fig. 8 in Mehlert et al. 1998) the age of the galaxy core of IC 4051 was determined as 12-17 Gyr, based on the Worthey SSP models (Worthey 1994). We note that agreement with model predictions was only obtained for the innermost $5{ }^{\prime \prime} 0$ of the galaxy core, for which the photometric data of the globular clusters suffer from contamination by the diffuse galaxy light. An additional error source is the fact that in the diffuse galaxy light the contributions of stellar sub-populations are blended and hard to disentangle.

The results of these $\chi^{2}$-tests are presented in Figs. 10 and 11 . The $\chi^{2}$-test results find the best fitting model to the age distribution in both GCSs to be a composite of an old population and a second population which is $\sim 10$ Gyr younger. In both galaxies, the best fitting model to the age distribution suggests approximately $60 \%$ to $70 \%$ intermediate age clusters. Such a surprisingly large fraction of young/intermediate globular clusters, also found in NGC 4365 and NGC 5846 (Hempel \& Kissler-Patig 2004), needs confirmation and careful investigation, especially since such large fractions of young stars should be visible in the integrated light, but are not detected yet. In fact, previous investigations based on optical photometry (Woodworth \& Harris 2000), suggest the bulk of the GCS being formed in situ, which, in the authors understanding, also includes the possibility of merging of almost completely gaseous objects. A late gaseous merger is not in any way ruled out using as well the argument of the central co-rotating disk and its very high metallicity.

The major goal in this work is to apply a semi-numerical method for detecting globular cluster sub-populations based on optical and near-infrared colors. As described in Hempel et al. (2003) and Hempel \& Kissler-Patig (2004) this procedure has been developed in order to partly compensate for the photometric uncertainties, increased by the usage of nearinfrared observations, when deriving age constraints. Instead of a direct comparison between the colors of each individual observed globular cluster with model predictions, as attempted with color-color diagrams (see Figs. 3 and 6) we rather look at the density of GCs with respect to a set of model isochrones and compare it to simulated GCSs. Although the results of

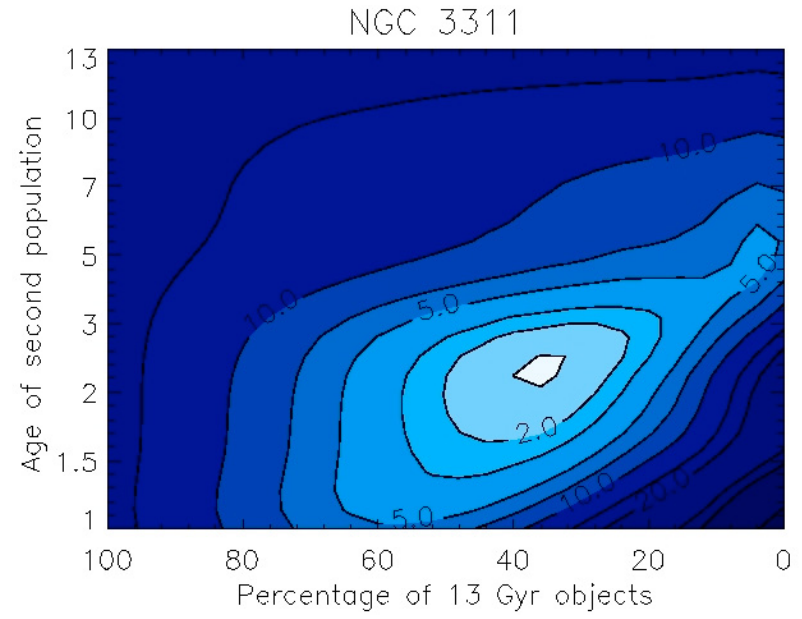

Fig. 10. $\chi^{2}$-test result for NGC 3311. Different levels represent the reduced $\chi^{2}$ of the comparison between the cumulative age distribution in NGC 3311 and various models. The best fitting model consists of $\sim 60 \%$ intermediate age globular clusters and $\sim 40 \%$ of $13 \mathrm{Gyr}$ old objects. The large size of this young population is surprising and is discussed in Sect. 5.

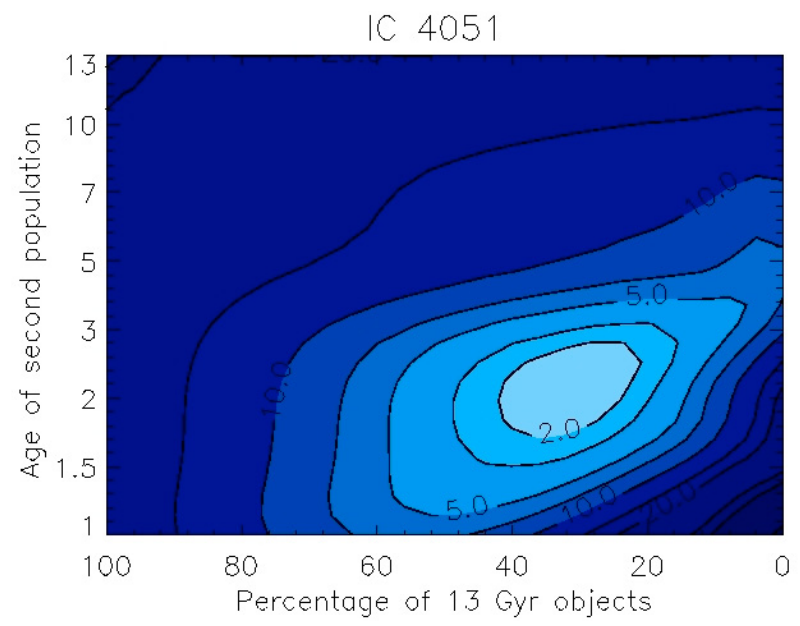

Fig. 11. The comparison between the age distribution in IC 4051 and the simulated systems again (as in Fig. 10) reveals a large fraction of young/intermediate age objects $(>\sim 60 \%)$, with the population of second generation globular clusters slightly larger than in NGC 3311.

both approaches may differ in the actual age estimates, the existence of distinct age sub-populations should be revealed by both approaches, the semi-numerical one being less sensitive to photometric errors. Again, concentrating on the IC 4051 results shown in the color-color diagram (see Fig. 3) we are unable to derive individual globular cluster ages by comparing the GC colors directly with the SSP model predictions, but nevertheless find a large fraction of globular clusters with $(V-I)$ colors being better represented by a isochrone for a 5 Gyr population or even younger. A better agreement between the age estimate via our semi-numerical approach should be possible by refining the age grid for the Monte-Carlo simulations (e.g. Hempel \& Kissler-Patig 2004) as well as for the determination of the cumulative age distribution. 
The derived age structure will therefore be affected by different factors, the most important being stated below:

- Firstly, we have to consider that the sample used in our analysis is color-selected, so only objects with $2.5 \leq(\mathrm{V}-$ $H) \leq 3.5$ and $0.6 \leq(V-I) \leq 1.4$ are used. The lower limit to $(V-H)$ is set due to the small color differences in the SSP models. The blue, metal-poor population of globular clusters will mostly contribute to the old sub-population and change the size ratio between old and young population in favor of the first generation objects. The upper limit, as well as the $(V-I)$ interval, is set to avoid significant contamination of our sample with background galaxies (Puzia et al. 2002). Especially the $(V-H)$ blue fraction, supposedly old globular clusters, will affect this result and shift the ratio between old and young/intermediate age globular clusters to higher values. However, as shown in Fig. 9, even in the extreme case where all GCs bluer than $(V-H)=2.5$ are old, 13 Gyr old clusters there remains a substantial fraction of young/intermediate age clusters.

- Secondly, we have to be aware of spatial bias effects. Due to the small NICMOS field of view, centered on the galaxy, the data cover only the innermost region of NGC 3311 and IC 4051. As observed by Schweizer \& Seitzer (1998) and simulated by Hibbard \& Mihos (1995) for the merger remnant NGC 7252, the central region of a galaxy is where one would expect to preferentially find the second generation of globular clusters, if they are indeed formed during a merger or accretion event. Although mostly born in the tidal tails (Li et al. 2004) of a merger, these star clusters will funnel toward the center of the merger remnant within several 100 Myr up to 1 Gyr (Hibbard \& Mihos 1995). Our samples are therefore spatially biased and the fraction of young/intermediate age globulars should be overestimated, compared to the global fraction over the entire galaxy.

- Thirdly, small number statistics will affect the $\chi^{2}$-test. As shown in Hempel \& Kissler-Patig (2004) the results of this test become unstable (i.e. show multiple best fitting age/size combinations) for small data sets. Previous tests have shown that $\gtrsim 100$ objects are needed to obtain reliable results. Strictly speaking this condition is only (barely) achieved in the IC 4051 sample. Recall that the total sample of IC 4051 and NGC 3311 contain 256 and 148 objects, respectively, but after applying the selection criteria (photometric error and color cuts) only 101 (IC 4051) and 70 (NGC 3311) objects contribute to the age distributions. Although the presence of a significant population of young/intermediate age globular clusters in NGC 3311 agrees with various other features of the galaxy (e.g. central dust lane) more data are required for a solid argument.

- Fourthly, as mentioned in Sect. 1 the SSP model isochrones by Bruzual \& Charlot (2003) have been applied. As shown by Hempel et al. (2003) and Hempel \& Kissler-Patig (2004) the color predictions for a given age by various SSP models differ particularly in the red color range and model dependencies should be considered and we will do so in detail in Appendix A. Since our method is based on the object density in color-color diagrams with respect to a given set of SSP isochrones, the final result, i.e. the detection of age sub-populations will hold independently of the SSP model. The number of objects required for a stable result of the $\chi^{2}$-test is however strognly dependent on the $\delta$ age $/ \delta(V-I)$ gradients. The models by Maraston (2005) are somewhat hampered by the fact that stellar populations with ages below 5 Gyr (below 2 Gyr for the 2005 release) show a non-monotonic increase of $(V-I)$ with $(V-H)$ relation, which leads to confusion in our counting algorithm. The major advance in the more recent SSP models are the inclusion of a well developed AGB phase (e.g. Renzini 1981; Maraston et al. 2001b), the possibility of a different $[\alpha / \mathrm{Fe}]$-enhancement (Thomas \& Maraston 2003) and the structure of the horizontal branch (Maraston 2005), resulting in color shifts. Although $\alpha$-enhancement seems to be more important when objects with solar metallicity are investigated no solid conclusions can be drawn, due to less solid calibration of the models. We will include the latest release of the Maraston models (2005), targeting the influence of the horizontal branch structure in an additional section (see Appendix A).

\section{Metallicity distribution}

In the framework of galaxy formation and evolution studies the age structure of the galaxy and its GCS plays a major role hence our main interest is focused on the age structure. Nevertheless, since the latter is determined by using SSP model isochrones, which combine age and metallicity predictions, it is necessary to test to what degree age and metallicity effects are still entangled. In the following we present the results of rough metallicity estimates based on the derived age structure.

A 2 Gyr old stellar population, with a $(V-H)$ color between $2.5 \leq(V-H) \leq 3.5$, i.e. consistent with the color limits set for the determination of the age distribution, contains objects with metallicities between $[\mathrm{Fe} / \mathrm{H}]=+0.00$ and $[\mathrm{Fe} / \mathrm{H}]=+1.1$, respectively, as derived via linear interpolation using the SSP models. Assuming a mean color of $(V-H)=3.0$ we obtain $[\mathrm{Fe} / \mathrm{H}]=+0.56$. Although, as shown in Fig. 11, the best fitting model consists of a 2 Gyr old second globular cluster population, the age uncertainty allows as well a higher age, i.e. $\sim 5$ Gyr. In this case the metallicity of objects in the same color range lies between $[\mathrm{Fe} / \mathrm{H}]=-0.2$ and +0.9 , with a mean value of $[\mathrm{Fe} / \mathrm{H}]=+0.4$ for $(V-H)=3.0$. As we will show, this corresponds closely to the mean metallicities determined for the young/intermediate GCs in IC 4051. Since the metallicity depends quite strongly on the derived age (more than the other way around) and although we can detect age subpopulations and set constraints on the age, the age uncertainty allows a large variety of metallicities. The following discussion about the metallicity distribution has to be seen in the light of this persistent degeneracy between age and metallicity.

With the introduction of combined optical and nearinfrared observations we are able to investigate the metallicity distribution approximately independently from the age structure, with the above caveats. In order to derive globular cluster metallicities we use the above-derived information about the age structure in the observed globular cluster 

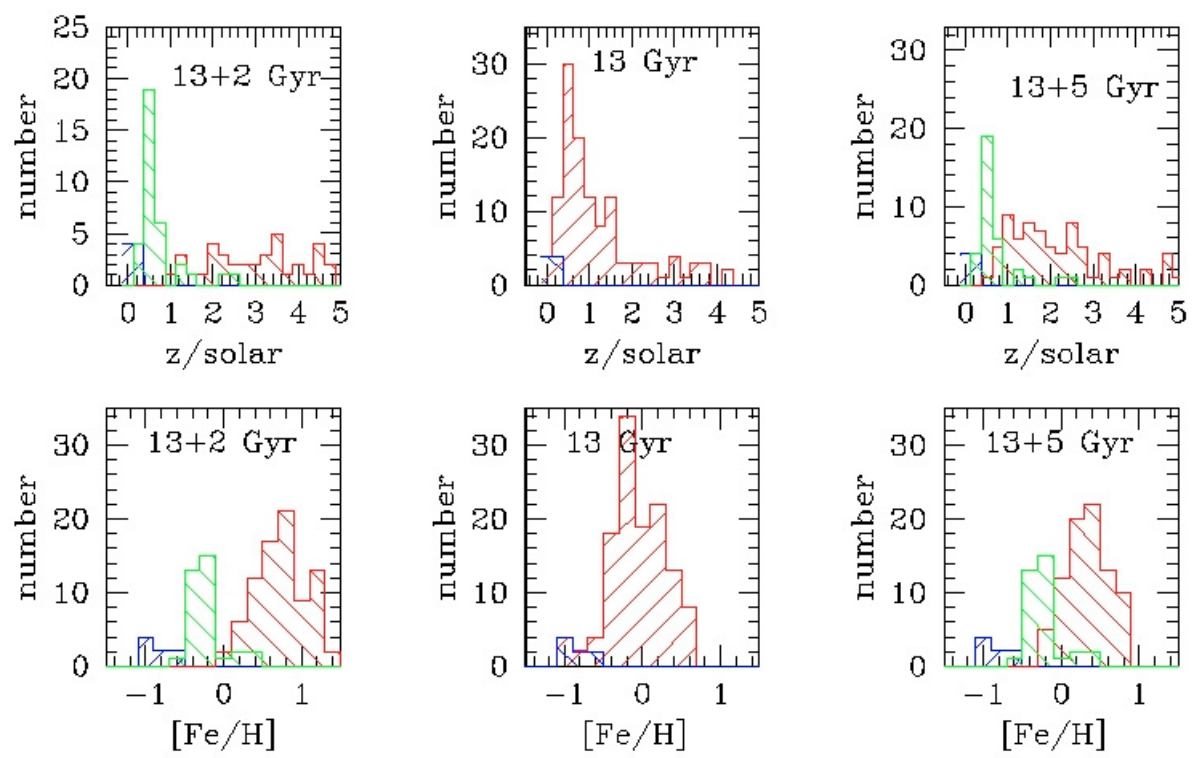

Fig. 12. Metallicity distribution in IC 4051 assuming different GC distributions. We show the metallicity distribution both in terms of linear metal abundance (upper panel) as well as in logarithmic metallicity $[\mathrm{Fe} / \mathrm{H}]$ (lower panels). In the left panels the metallicity distribution is based on the assumption of a 2 and $13 \mathrm{Gyr}$ old population (following the best fit model from the age distribution), whereas the central panel assumes all clusters to be $13 \mathrm{Gyr}$ old. We also show the metallicity distribution assuming the young/intermediate globular clusters to be 5 Gyr old (right panel). The color coding of the histograms is as follows; red: young and metal rich, green: old and metal rich, blue: old and metal-poor.

sample (see Figs. 10 and 11). The results in Sect. 3.2 tell us that the age structure in both systems is well matched by a mix of a 13 Gyr old population with globular clusters which are $\sim 10$ Gyr younger. The determination of the metallicity distribution follows the procedure given below and applies the Bruzual \& Charlot (2003) SSP model isochrones.

- We split the globular cluster sample (selected by photometric error) with respect to the $(V-H)$ color into a blue $((V-H)<2.5)$ and presumably old population and a second, red $(2.5<(V-H))$ sub-population, containing both old as well as young/intermediate globular clusters. In addition we set an upper limit of $(V-H) \leq 3.5$ to avoid strong contamination with background galaxies (Hempel \& Kissler-Patig 2004).

- For globular clusters found in the blue sub-population, the metallicities (given as $Z / Z_{\odot}$ ) were calculated by interpolating the metallicity- $(V-H)$ correlation of the 13 Gyr model isochrone.

- Corresponding to the age structure, we found that the red sub-population is split into old and intermediate/young populations, and use the 5 Gyr model isochrone for the latter. All objects with $(V-I) \leq(V-I)_{5 \text { Gyr }}$ are then assumed to form the second generation of globular clusters. Although the comparison of the cumulative age distributions to simulated GCSs assigns the intermediate/young objects to an age of $\sim 2 \mathrm{Gyr}$, we allow an age uncertainty of $3 \mathrm{Gyr}$, based on the $\chi^{2}$ contours, and set the age split at 5 Gyr alternatively.

- The metallicity is then determined by interpolating $Z / Z_{\odot}$ at $(V-H)_{G C}$ using the $13 \mathrm{Gyr}$ and $2 \mathrm{Gyr}$ isochrones (Figs. 12 and 13, left panels). For comparison we also derive the metallicity distributions assuming a purely old globular cluster population (Figs. 12 and 13, middle panels) and for mixed GCSs containing 13 Gyr and 5 Gyr old objects (Figs. 12 and 13, right panels). In both figures the upper panels give the metallicities on a linear scale, whereas the lower panels refer to $[\mathrm{Fe} / \mathrm{H}]$ values.

- The metallicity estimates depend strongly on the age structure and are therefore also hampered by bias effects (see Sect. 3.2). The case of NGC 3311 suffers from smallnumber statistics, which makes the results of the $\chi^{2}$-test statistically unstable. Nevertheless, the comparison of the ( $V-$ $H$ ) colors, seen in Figs. 3 and 7, shows that NGC 3311 lacks the most metal rich globular clusters found in IC 4051. Due to the above uncertainty for NGC 3311 we will concentrate on the GCS in IC 4051.

In the discussion about the bimodality of metallicity distributions we want to emphasize the importance of the chosen scaling. It is interesting to note that the metallicity distribution for a purely old (13 Gyr) population seems to suggest a bimodality in the $[\mathrm{Fe} / \mathrm{H}]$ distribution for the IC $4051 \mathrm{GC}$, which is NOT seen if we transform the GC metallicities into linear abundances. However, if we fit the $[\mathrm{Fe} / \mathrm{H}]$ distribution by either a double or a single Gaussian, as shown in the middle panels in Fig. 14, the resulting $\chi^{2}$ of the fit is found to be $\chi^{2}=0.6$ and 0.8 for the double and single Gaussian, respectively, virtually identical. Hence there is no strong evidence for bimodality in the metallicity distribution, which considers all clusters as old. Although a bimodal distribution is not favored over unimodality in the 13 Gyr old population, it becomes obvious in the samples assuming mixed globular cluster age distributions (see upper and lower panel in Fig. 14).

Since we find objects within a wide range of metallicities, applying a logarithmic scaling, as given in the distribution of 

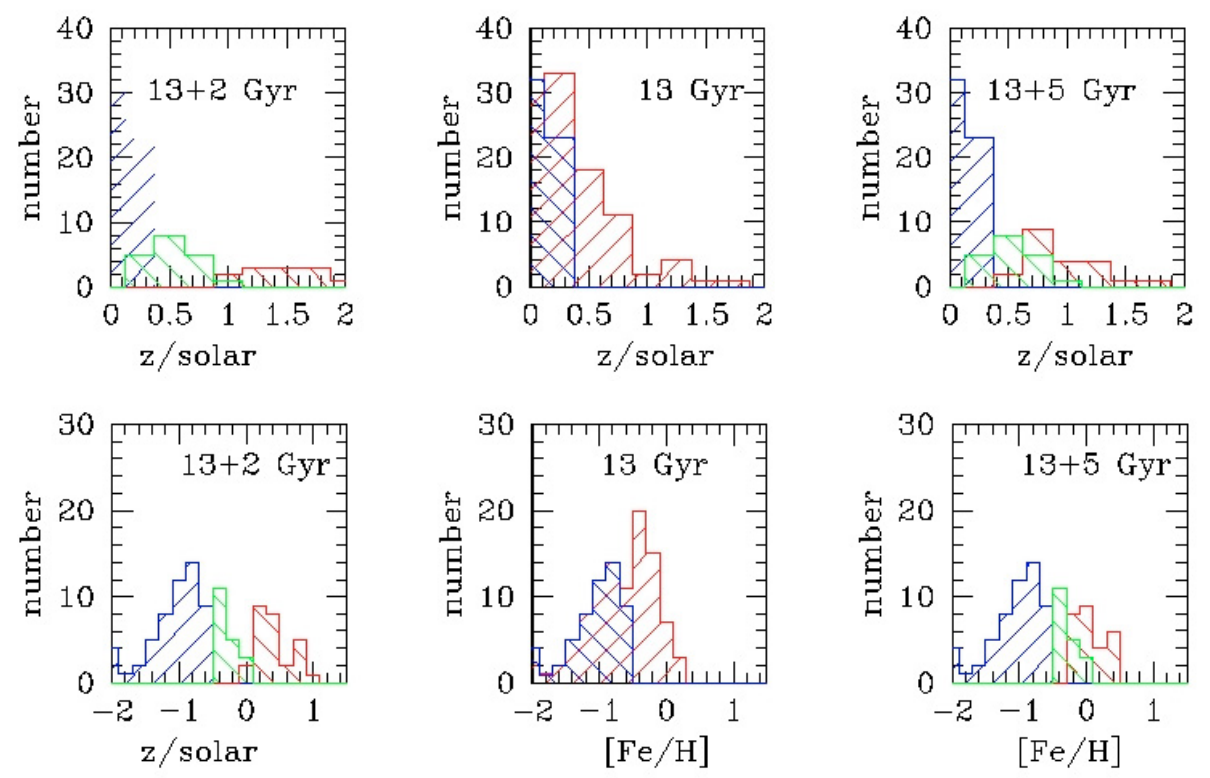

Fig. 13. Metallicity distribution in NGC 3311 assuming different age distributions. We apply the same color coding and method for displaying the abundance distribution as in Fig. 12.

the $[\mathrm{Fe} / \mathrm{H}]$ values, increases the chances for detecting metallicity populations. In the linear $\mathrm{Z}$ space formerly clearly detected $[\mathrm{Fe} / \mathrm{H}]$-populations are much more spread out and may not be detected anymore, e.g. in smaller samples or in globular cluster populations with a smaller age difference. The metallicity distribution given in $Z / Z_{\odot}$ for a $13+2$ or 5 Gyr old population is widely spread. We caution that a bimodal color distribution does not necessarily translate into a clear bimodal metallicity or linear abundance distribution (see also Kissler-Patig 2002).

The mean metallicity based on the $(V-I)$ colors alone and a unimodal color distribution (Woodworth \& Harris 2000) was determined to be $\langle\mathrm{Fe} / \mathrm{H}\rangle=-0.3$ or $Z / Z_{\odot}=0.5$. Working with the more metal sensitive $(V-H)$ color we obtain the following results. Assuming a purely 13 Gyr old population in IC 4051 (see Fig. 12, central panel), we derive a mean metallicity of $\langle\mathrm{Fe} / \mathrm{H}\rangle \sim-0.1$, assuming a unimodal distribution of the metallicity. For metal-poor AND a metal rich-population of the same age we obtain mean metallicities of -0.27 and +0.12 dex, respectively. For a mixed-age GCS, the mean metallicity of the 2 Gyr old population is derived as $\langle\mathrm{Fe} / \mathrm{H}\rangle_{2 \mathrm{Gyr}}=+0.7$, with $\langle\mathrm{Fe} / \mathrm{H}\rangle_{13 \mathrm{Gyr}}=-0.9$ and -0.3 for the metal-poor and metalrich old population. Assuming $5 \mathrm{Gyr}$ as the age of the intermediate age population, the results change only marginally to $\langle\mathrm{Fe} / \mathrm{H}\rangle_{5 \text { Gyr }}=+0.34$. The metallicities derived for the young metal-rich populations in either case are very high; compared to observed spectroscopic metallicities for other second generation GCs (e.g. Schweizer \& Seitzer 1998; Goudfrooij et al. $2001 \mathrm{a}, \mathrm{b})$ and must be regarded as very tentative. Several issues have to be discussed here, which all affect the derived metallicity distribution.

- Again, the basic requisite to derive globular cluster metallicities is an accurate age determination. Although the combined optical and near-infrared photometry allows us to derive the mean age of sub-populations, the age-metallicity degeneracy is not entirely broken. Even more important, for a given $(V-H)$-bin the derived metallicity depends on the SSP isochrone. Thus applying the mean age of suppopulations to calculate individual metallicities causes additional systematic errors.

- In our analysis we use the SSP model isochrones by Bruzual \& Charlot (2003). If we compare the color predictions of these models with Vazdekis (1999) or Maraston et al. (2001a) then we find considerable differences, especially within the red populations. Following the Bruzual \& Charlot models a 5 Gyr old object would by $\sim 0.1$ mag redder in $(V-I)$ compared to the Vazdekis models, given the same $(V-H)$. This discrepancy has only little effect on the mean ages of globular cluster sub-populations as long as we apply identical SSP models for the age determination in observed and simulated samples and do not attempt to derive ages of individual clusters. Therefore, our primary result of a significant number of intermediate age globular clusters will not change. The fact that its age alters with the applied SSP models between 2 and 5 Gyrs is of no relevance since the method itself does not allow more precise age estimates. To quantify the effect of our choice of SSP models on the metallicity distribution we will repeat the analysis, i.e. the determination of the cumulative age distribution and the modeling procedure, using the SSP model isochrones by Maraston (2005) (see Appendix A).

- In Paper IV of this series (Hempel \& Kissler-Patig 2004) the contamination of the globular cluster sample with unresolved background galaxies has been discussed. Although we try to limit the contamination by setting limits on $(V-H)$ and $(V-I)$ we can not entirely exclude it. These color limits are derived from the SSP model isochrones, assuming a maximum GC age of 15 Gyr and a maximum metallicity of $2.5 \times Z_{\odot}$ for the younger generation of GCs.

As already mentioned, both GCSs have been discussed previously (e.g. Woodworth \& Harris 2000; Brodie et al. 2000) and 

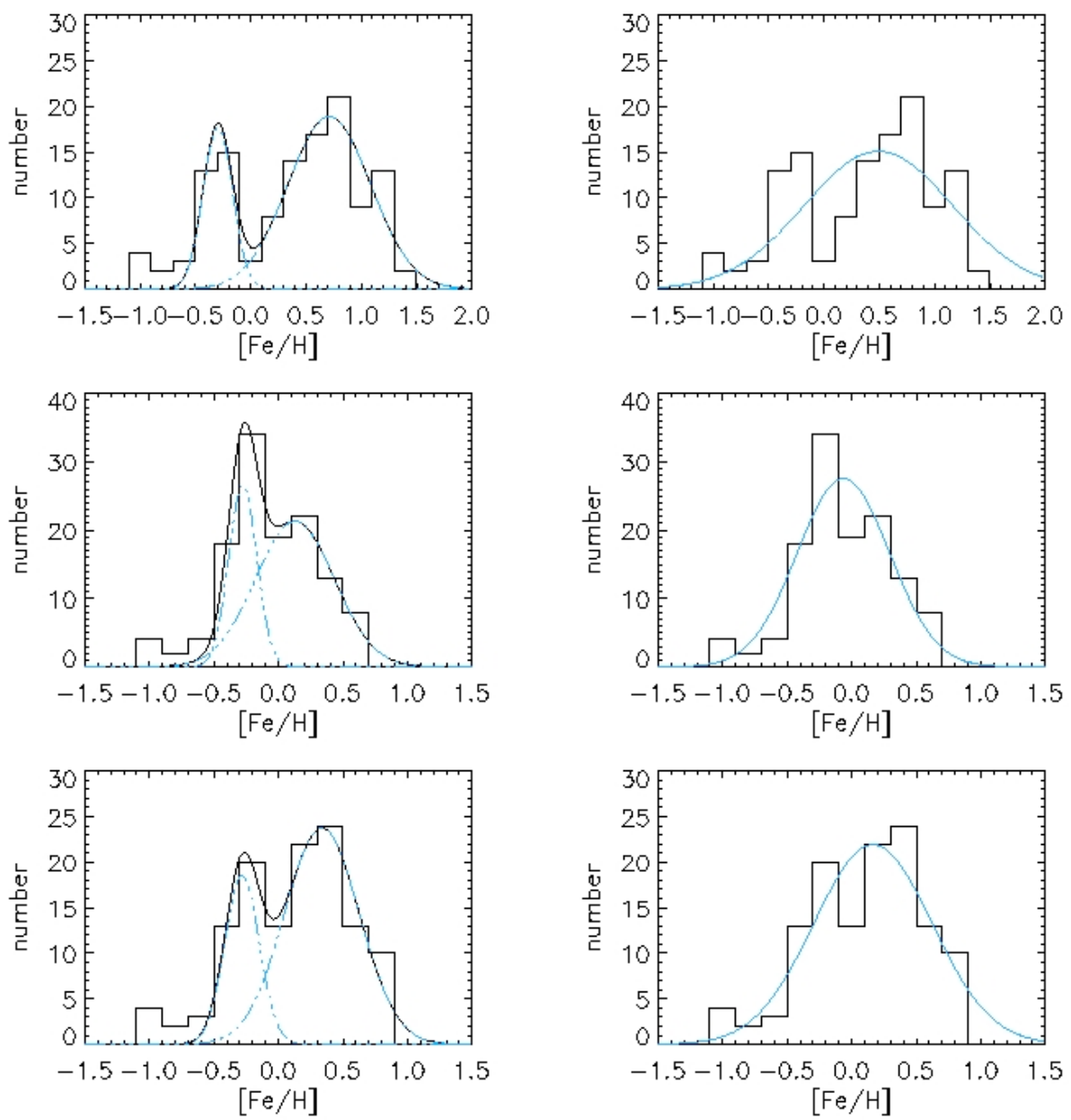

Fig. 14. Metallicity distribution in IC 4051 assuming a combination of a 13 and 2 Gyr old population (upper panels), a pure 13 Gyr old population (middle panels) and a $13+5 \mathrm{Gyr}$ old mixed population (lower panels). On the left we show the best bimodal Gaussian fit while on the right that for a single Gaussian. Clearly the mixed populations are best fitted by a double Gaussian (dashed-dotted lines). The fit to the metallicity distribution of a $13 \mathrm{Gyr}$ population gives similarly good results for a single and a double Gaussian, although the $\chi^{2}$ of the fit is slightly better for a single Gaussian. In the left panel the dashed-dotted lines shows the two Gaussians whereas the solid line shows their combination.

to further investigate our claims a direct comparison with previous studies is useful. However, in the case of NGC 3311 this is only possible in a more general way since the study by Brodie et al. (2000), building on the same optical data, includes only detections on the wide-field chips of HST/WFPC2, whereas combined optical and near-infrared data are only available for the innermost region, covered by the PC chip.

In IC 4051 the $(V-I)$ distribution for objects which are later also detected in the infrared, is best fitted with a single Gaussian centered on $V-I=1.17 \pm 0.005$ (see Fig. 15, left panel), which is though somewhat redder, due to color selection, but still in agreement with the results given by Woodworth $\&$ Harris $(2000)(V-I)=1.106$. Using the linear conversion by Woodworth \& Harris (2000): $V-I=0.17[\mathrm{Fe} / \mathrm{H}]+1.15$ the mean $(V-I)$ colors translate into mean metallicities of $\approx+0.11$ and $\approx-0.3$, respectively. We note again that these values refer to different samples, based on the observed field of view.

In the discussion about the metallicity distribution for the NGC 3311 GCS we have to be especially cautious given the above caveats about the dependence of the metallicity distribution on the derived ages and is included rather for completeness than for solid argumentation. Comparing the distribution of $(V-I)$ color, as shown in Fig. 15 (right panel), with the results obtained for the wide-field chip detections, reveals as well a bimodal color distribution with the center of the blue and red sub-population at $0.91 \pm 0.01 \mathrm{mag}$ and $1.11 \pm 0.01 \mathrm{mag}$, respectively. In comparison, in the work by Brodie et al. (2000) the $(V-I)$ color distribution peaks at $0.91 \pm 0.03 \mathrm{mag}$ and $1.09 \pm 0.03 \mathrm{mag}$, resulting in a metallicity of $[\mathrm{Fe} / \mathrm{H}] \approx-1.5$ and $\approx-0.75 \mathrm{dex}$. The color-metallicity conversion follows the relation given in Kissler-Patig et al. (1998): $[\mathrm{Fe} / \mathrm{H}]=(-4.50 \pm 0.30)+(3.27 \pm 0.32)(V-I) . \mathrm{In}$ contrast, the metallicities presented in this work, derived from the $(V-H)$ color directly, but are based on the estimated age and a subsequent application of the SSP model isochrones.

\section{Summary}

We have investigated the age structure of GCs in two luminous early-type galaxies, IC 4051 and NGC 3311, each with large GC populations, using the combination of optical and 

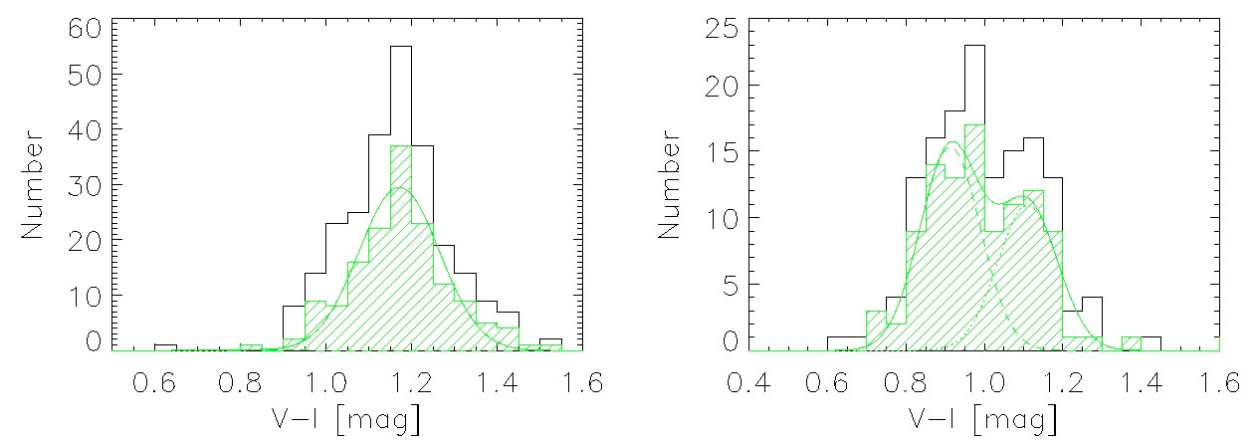

Fig. 15. $(V-I)$ color distribution for globular clusters in IC 4051 (left) and NGC 3311 (right) detected on the PC chip, for which as well $H$-band data are available. The open histogram represents the complete VIH sample, whereas the shaded histogram includes only objects for which the photometric errors, $\delta(V-I)$ and $\delta(V-H)$ have been derived to be $<0.15 \mathrm{mag}$. The solid curve shows either the single Gaussian (for IC 4051) or the sum of the two Gaussian functions (dotted and dashed lines) in case of NGC 3311.

near IR colors to help break the age-metallicity degeneracy. In both galaxies, we find evidence for a significant fraction of young/intermediate age clusters $(\approx 2-5 \mathrm{Gyr})$, in addition to their old counterparts, by comparing the observed color distribution with models. This result is confirmed by the comparison between the cumulative age distribution in observed and simulated systems, which not only indicates a second major cluster formation event but also allows to set some constraints on the time frame. Further evidence for a less passive evolution of the galaxies is given by independent features (dust lanes, co-rotating core), but especially in NGC 3311, the effects of small number statistics make our results tentative. Based on the small number of objects in this sample, our detected fraction of 50-60\% of young globular clusters in the total GCS in NGC 3311 has a large uncertainty. The results for IC 4051 are more statistically reliable, suggesting more than half of the globular clusters are young/intermediate age objects. This fraction should be regarded as an upper limit, considering that most of the GCs excluded from the age analysis are probably mainly old, and that our observations are biased to the central regions, where younger objects should preferentially be found. Thus, at this stage the global importance of the star forming event leading to a second generation of globular clusters in NGC 3311 and IC 4051 cannot be firmly established. More data, specially sprectroscopic, over a wider galactocentric field, would be useful for clarifying the nature of these populations. Nevertheless, the improved age resolution of combined optical and nearinfrared photometry reveals age sub-populations, which could not be detected using optical data alone.

Preceding the results presentet in Appendix A we want to include the effects of SSP model dependency in this summary. Although of little consequence for the age distribution the choice of the SSP model is becomes important for the derived metallicity distribution. The extremely high mean metallicity of the $2 \mathrm{Gyr}$ old population $([\mathrm{Fe} / \mathrm{H}] \approx 0.8 \mathrm{dex}$, see Sect. 4), obtained with the Bruzual \& Charlot (2003) models, becomes much more reasonable when the Maraston (2005) are applied, i.e. $[\mathrm{Fe} / \mathrm{H}] \approx 0.2$. Whether this large discrepancy is based on the models themselves, with only the Maraston models including the TP-AGB phase, or is merely an artifact of the interpolation of the metallicity, or both, still needs to be investigated. Nevertheless we note that the difference in metallicity decreases with an inceasing age of the cluster population. Due to the small number of metal-poor objects we do not discuss model dependencies for this sub-sample here.

Acknowledgements. Support for this work was provided by NASA through grant number GO-07280 from the Space Telescope Science Institute, which is operated by the Association of Universities for Research in Astronomy, Inc., under NASA contract NAS5-26555. K. Mighell kindly provided advice concerning the CCDCAP package. D.G. would like to thank Nick Suntzeff and Malcolm Smith of the staff of Cerro Tololo Inter-American Observatory for their kind support of this project. D.G. gratefully acknowledges support from the Chilean Centro de Astrofísica FONDAP No. 15010003. The authors wish also to thank the anonymous referee for her/his comments, which helped immensely to improve the manuscript.

\section{References}

Ashman, D. M., \& Zepf, S. E. 1998, Globular Cluster Systems (Cambridge University Press)

Barmby, P., Huchra, J. P., Brodie, J. P., et al. 2000, AJ, 119, 727

Baum, W. A., Hammergren, M., Thomsen, B., et al. 1997, AJ, 113, 1483

Bertin, E., \& Arnout, S. 1996, A\&AS, 117, 393B

Brodie, J. P., Larsen, S., \& Kissler-Patig, M. 2000, ApJ, 543, L19

Brodie, J. P., Strader, J., Denicoló, G., et al. 2005, AJ, in press [arXiv:astro-ph/0502467]

Bruzual, G. A., \& Charlot, S. 1993, AJ, 405, 538

Bruzual, G. A. 2000, private communication

Bruzual, G. A., \& Charlot, S. 2003, MNRAS, 344, 1000

Cohen, J. G., \& Matthew, K. 1994, AJ, 108, 128

Davies, R. L., Kuntschner, H., Emsellem, E., et al. 2001, AJ, 548, 33

deVaucoleurs, G., deVaucouleurs, A., Corwin, H. G., et al. 1991, Third Ref.Catalogue of Bright Galaxies (New York: Springer)

Dickinson, M., Sosey, M., Rieke, M., et al. 2002, NICMOS Photometric Calibration, in the proceedings of the 2002 HST Calibration Workshop, Space Telescope Science Institute, Baltimore, Maryland, 17./18.10. 2002, ed. S. Arribas, A. Koekemoer, \& B. Whitmore, 233

Ferrari, F., Pastoriza, M. G., Macchetto, F., et al. 1999, A\&AS, 136, 269

Gebhardt, K., \& Kissler-Patig, M. 1999, AJ, 118, 1526

Geisler, D., Lee, M. G., \& Kim, E. 1996, AJ, 111, 1529

Goudfrooij, P., Mack, J., Kissler-Patig, M., et al. 2001, MNRAS, 322, 643 
Goudfrooij, P., Alonso, M. V., Maraston, C., et al. 2001, MNRAS, 328,237

Grillmair, C. J., Faber, S. M., Lauer, T. R., et al. 1994, AJ, 108, 102

Harris, W. E., \& van den Bergh, S. 1981, AJ, 85, 1627

Harris, W. E., Smith, M. G., \& Myra, E. S. 1983, ApJ, 272, 456

Harris, W. E. 1991, ARA\&A, 29, 543

Harris, W. E. 2001, in Star Clusters, Saas-Fee Advanced Course 28, Swiss Society for Astrophysics and Astronomy, ed. L. Labhardt, \& B. Binggeli (Berlin: Springer-Verlag), 223

Hempel, M., Hilker, M., Kissler-Patig, M., et al. 2003, A\&A, 405, 487, Paper III

Hempel, M., \& Kissler-Patig, M. 2004, A\&A, 419, 863, Paper IV

Hempel, M. 2004, Ph.D. Thesis, Early-Type Galaxies and Their Sometimes Not So Old Globular Clusters, Ludwig-Maximilian'sUniversity Munich

Hibbard, J. E., \& Mihos, J. C. 1995, AJ, 110, 140

Hilker, M. 2002, in Extragalactic Star Clusters, ed. D. Geisler, E. K. Grebel, \& D. Minniti (San Francisco: ASP), Proc. IAU Symp., 207, 281

Hilker, M. 2003, in New Horizons in Globular Cluster Astronomy, ed. G. Piotto, G. Meylan, G. Djorgovski, \& M. Riello, ASP Conf. Ser., 296, 583

Hilker, M. 2003, in ESO Astrophysics Symposia, Extragalactic Globular Cluster Systems, ed. M. Kissler-Patig (Springer), 173

Holtzman, J. A., Burrows, C. J., Casertano, S., et al. 1995, AJ, 107, 1065

Jensen, J. B., Tonry, J. L., Thomson, R. I., et al. 2001, ApJ, 550, 503

Jorgensen, I., \& Franx, M. 1994, ApJ, 433, 553

Jura, M. 1986, ApJ, 306, 483

Kent, S. M., \& Gunn, J. E. 1982, AJ, 87, 945

Kissler-Patig, M., Brodie, J. P., Schroder, L. L., et al. 1998, AJ, 115, 105

Kissler-Patig, M. 2002, IAU Symp., 207, 207

Kissler-Patig, M., Brodie, J. B., \& Minniti, D. 2002, A\&A, 391, 441, Paper I

Kundu, A., \& Whitmore, B. C. 2001, AJ, 121, 2950

Kundu, A., \& Whitmore, B. C. 2001, AJ, 122, 1251

Kuntschner, H., Smith, R. J., Colless, M., et al. 2002, MNRAS, 337, 172

Larsen, S., Brodie, J. P., Beasley, M. A., et al. 2003, ApJ, 585, 767

Lee, H.-C., Lee, Y.-W., \& Gibson, B. K. 2002, A\&A, 124, 2664

Li, Y., Mac Low, M.-M., \& Klessen, R. S. 2004, ApJ, submitted [arXiv: astro-ph/0407248]
Malhotra, S. 2002, NICMOS Instrument Handbook, Version 5.0 (Baltimore: STScI)

Maraston, C., \& Thomas, D. 2000, ApJ, 541, 126

Maratson, C., Greggio, L., \& Thomas, D. 2001a, Ap\&SS, 276, 893

Maraston, C., Kissler-Patig, M., Brodie, J. P., et al. 2001b, A\&A, 370, 176

Maraston, C. 2005, MNRAS, submitted

McLaughlin, D. E., Secker, J., Harris, W. E., et al. 1995, AJ, 118, 1033

McNamara, B. R., Bregman, J. N., \& O’Connell, R. W. 1990, AJ, 360, 20

Mehlert, D., Saglia, R. P., Bender, R., et al. 1998, A\&A, 332, 33

Mehlert, D., Thomas, D., Saglia, R. P., et al. 2003, A\&A, 407, 423

Mighell, K. J., \& Rich, R. M. 1995, AJ, 110, 1649

Minniti, D., Alonso, M. V., Goudfrooij, P., et al. 1996, ApJ, 467, 221

Puzia, T. H., Zepf, S. E., Kissler-Patig, M., et al. 2002, A\&A, 391, 453, Paper II

Puzia, T. H., Kissler-Patig, M., Thomas, D., et al. 2004a, A\&A, 415, 123

Puzia, T. H., Kissler-Patig, M., Thomas, D., et al. 2004b, A\&A, submitted

Renzini, A. 1981, AJ, 94, 175

Richter, O.-G. 1989, A\&AS, 77, 237

Searle, L., \& Zinn, R. 1978, ApJ, 225, 357

Secker, J., Geisler, D., McLaughlin, D. E., et al. 1995, AJ, 109, 1019

Schlegel, D. A., Finkbeiner, D. P., \& Davis, M. 1998, ApJ, 500, 525

Schweizer, F., \& Seitzer P. 1998, AJ, 116, 2206

Stephens, A. W., Frogel, J. A., Ortolani, S., et al. 2000, AJ, 119, 419

Thomas, D., \& Maratson, C. 2003, A\&A, 401, 429

Thomas, D., Maraston, C., \& Bender, R. 2003, MNRAS, 339, 897

Tully, B. 1988, Nearby galaxies catalog (Cambridge University Press)

Västerberg, A. R., Jörsäter, S., \& Lindblad, P. O. 1991, A\&A, 247, 335

Vazdekis, A. 1999, ApJ, 513, 224

Vazdekis, A., Cenarro, A. J., Gorgas, J., et al. 2003, MNRAS, 340, 1317

Whitmore, B. C., \& Schweizer, F. 1995, AJ

Wielen, R. (ed.) 1990, Dynamics and Interactions of Galaxies (Springer Verlag)

Woodworth, S. C., \& Harris, W. E. 2000, AJ, 119, 2699

Worthey, G. 1994, ApJS, 95, 107

de Zeeuw, P. T., Bureau, M., Emsellem, E., et al. 2002, MNRAS, 329, 513

Zepf, S., \& Ashman, D. 1993, MNRAS, 264, 611 
M. Hempel et al.: Extragalactic globular clusters in the near infrared. V., Online Material p 1

\section{Online Material}




\section{Appendix A: SSP model dependency}

In the recent past, tremendous progress has been made in the understanding of stellar evolution and one of the major outcomes are more and more accurate models of how colors and spectral features for single stellar populations evolve in time, depending on metallicity and other parameters, e.g. the structure of the horizontal branch (e.g. Maraston \& Thomas 2000; Lee et al. 2002). Although this paper does not intend to argue in favor of a specific SSP model we would like to show how the results depend on our choice of the SSP model. In the main part of this paper we apply the new version of the Bruzual \& Charlot model isochrones (2003). As shown in Hempel \& Kissler-Patig (2004) and Hempel (2004) the choice between the Bruzual \& Charlot models $(2000,2003)$ or, alternatively, the model by Vazdekis (1999) or Maraston (2001) affect the results only marginally, i.e. the detection of intermediate age clusters is essentially independent of the selected model. However, in order to investigate the subsequent advances made in SSP modeling we will as well conduct our analysis applying the latest version of the SSP models by Maraston (2005), assuming a red/intermediate horizontal branch structure and a Salpeter IMF. The $(V-I)$ and $(V-H)$ colors for both the Bruzual \& Charlot (2003) and Maraston (2005) model are compared in Fig. A.1. We note that unlike earlier releases of both SSP models the color predictions for a given age do not differ significantly, except for metal-poor objects with ages $\leq 2$ Gyr. Prior to our analysis we compared as well the model predictions for the $(V-I)$ and $(V-H)$ color assuming a red/intermediate and a blue horizontal branch and various ages ( $>1 \mathrm{Gyr}$ ) as offered by the SSP models. With the exception of a $1 \mathrm{Gyr}$ old stellar population, the change in color is smaller than the photometric error in the observational data and in fact smaller than the color difference between models using different IMF's, especially within the red $(V-H)$ color range where our technique is sensitive to the detection of young/intermediate GCs.

The color-color diagrams, for both globular cluster systems are again shown in Figs. A.2 and A.3. In contrast to Figs. 3 and 7 the colors are now compared to the SSP models by Maraston (2005). Still the color distributions show a large fraction of globular clusters with a $(V-I)$ color which is too blue to be consistent with a population older than $5 \mathrm{Gyr}$.

In order to detect GC sub-populations within a galaxy we derive the cumulative age distribution (see Hempel et al. 2003; Hempel \& Kissler-Patig 2004) based on optical and nearinfrared colors in the same way as in Sect. 3.2. Hereby the GC density with respect to the model isochrones is of more importance than the color of each GC separately. As described before, the GC density is determined as the number of objects with a $(V-I)$ color redder and therefore older than a given isochrone. Previously those number counts were estimated for the $1,2,3,5,7,10,13$ and $15 \mathrm{Gyr}$ isochrone. Using the new Maraston models requires a small alteration in this procedure. Despite the non-monotonic pattern of the $1 \mathrm{Gyr}$ isochrone we follow the same counting procedure, taking advantage of the fact that, independent from metallicity, the model predictions for $(V-I)$ give a redder color for a 2 Gyr old population
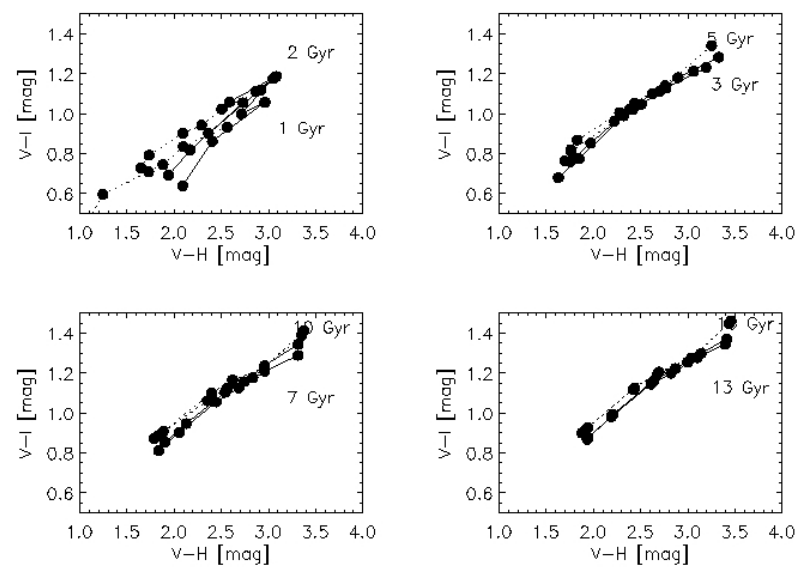

Fig. A.1. Comparison between the color predictions of the Bruzual \& Charlot (2003, dotted line) and the Maraston (2005, solid line) SSP models. Clearly the largest discrepancy is found for objects younger than $3 \mathrm{Gyr}$, whereas for older populations the difference in $(V-I)$ is in the range of the photometric error or below. We note that the models are given for different metallicities (Bruzual \& Charlot: $-2.25,-1.35,-0.64,-0.33,0.0,+0.56 \mathrm{dex}$; Maraston: -2.25 , $-1.35,-0.33,0.0,+0.35$ dex $)$.

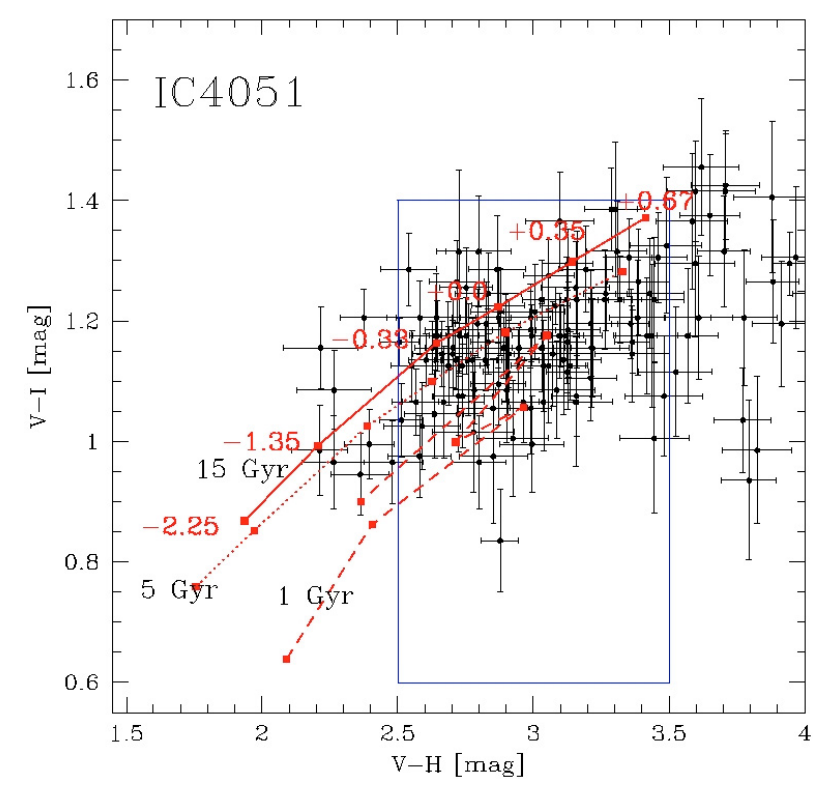

Fig. A.2. $(V-I)$ vs. $(V-H)$ color-color diagram for IC 4051. As in Fig. 3 only GCs with photometric errors $<0.15$ mag have been selected. The 1, 5, and $15 \mathrm{Gyr}$ isochrones are marked as solid, dotted and dashed line, respectively. Here the SSP model by Maraston has been applied.

compared to $1 \mathrm{Gyr}$ population. Nevertheless, in the simulations we assume the second generation of GC to be at least 2 Gyr old.

Comparing the cumulative age distributions in both observed systems with our set of simulations, shows at first significant differences, if the Maraston SSP models are applied (Figs. A.4 and A.5). Hence we need to emphasize again that this approach does not aim at high precision age estimates but is rather a tool to detect significant sub-populations of GCs. Ideally, a successful detection should be complemented by spectroscopic investigations to determine the age more 
M. Hempel et al.: Extragalactic globular clusters in the near infrared. V., Online Material p 3

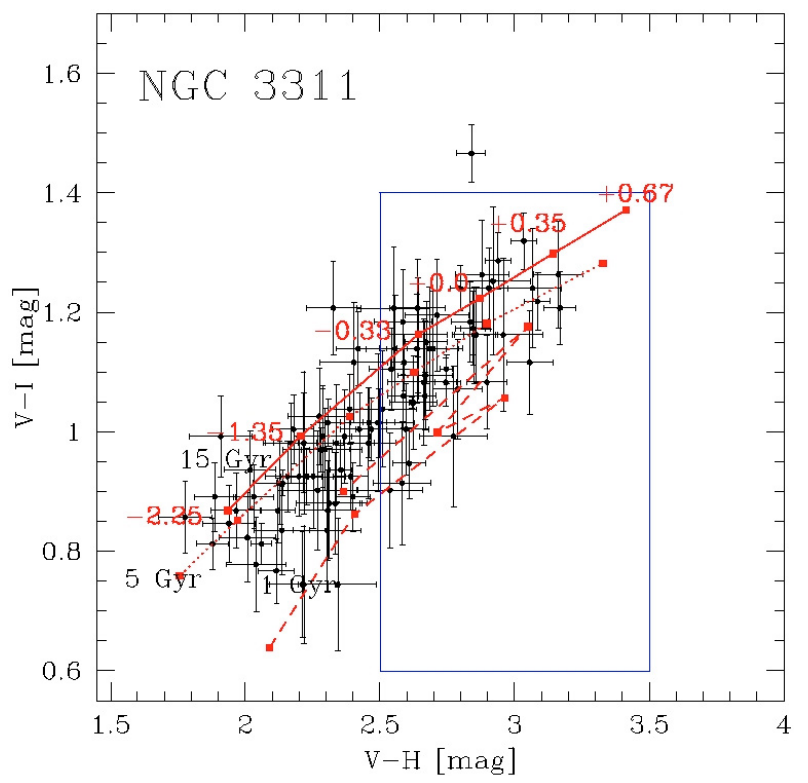

Fig. A.3. $(V-I)$ vs. $(V-H)$ color-color diagram for NGC 3311. As in Fig. 3 only GCs with photometric errors $<0.15$ mag have been selected. The 1,5, and $15 \mathrm{Gyr}$ isochrones are marked as solid, dotted and dashed line, respectively. Here the SSP model by Maraston has been applied.

accurately. Due to the large distance of most early-type galaxies (especially in high density environment) spectroscopic surveys are hardly feasible. Keeping this in mind we find that the conclusions about a second population of intermediate age globular clusters with the selected sample is confirmed, although the relative size of the second GC generation is shifted to larger values, i.e. using the Maraston (2005) models leads to an even larger fraction of intermediate age clusters. Since the latter is affected by various biases (color cuts, spatial bias, etc.) we consider large numbers of intermediate/young GCs to be an upper limit at best. Considering the importance of horizontal branch structure for the modeling of SSP isochrones this result seems to be surprising. On the other side we have to be aware that a major impact of the HB structure is expected for the colors of metal-poor objects (see Fig. A.1), which we exclude from our analysis by setting color cuts.

To round things up we will finish this Section by deriving the metallicity distribution in IC 4051, following the procedure described in Sect. 4, but now based on the Maraston (2005) models. As before we calculate the metallicity under the assumption of the globular clusters being 2, 5, or 13 Gyr old, depending on their $(V-I)$ color with respect to the corresponding isochrones. The metallicity $[\mathrm{Fe} / \mathrm{H}]$ for each objects is then interpolated for a given $(V-H)$.

Comparing the color predictions of both models we note that stellar populations older than $3 \mathrm{Gyr}$ are almost unaffected, the Bruzual \& Charlot $(V-I)$ colors being somewhat bluer than their Maraston counterparts. Translated into ages the globular clusters are slightly younger when the Maraston models are applied. There is, however, a significant difference in $(V-H)$ with respect to the corresponding metallicity values. As shown in Fig. A.1 the $(V-H)$ color of a 1 or 2 Gyr old population of low

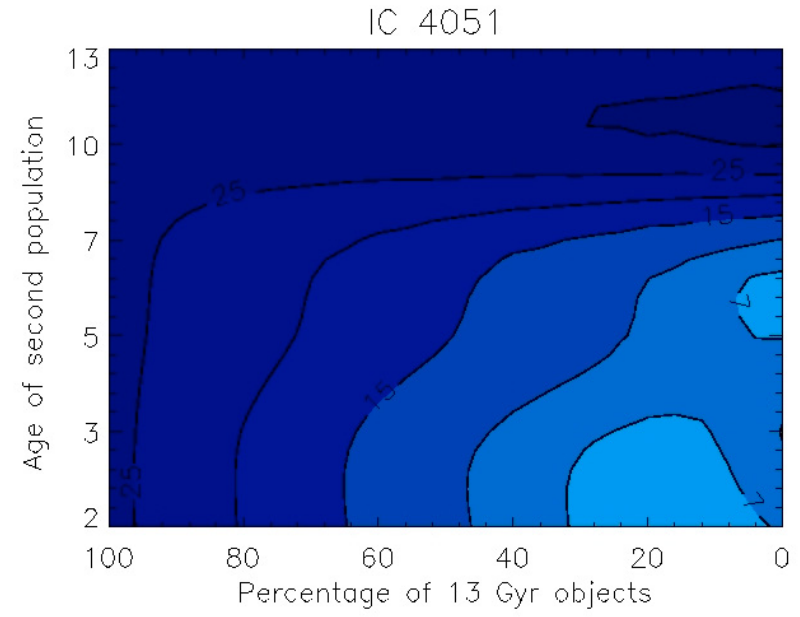

Fig. A.4. $\chi^{2}$-test result for IC 4051. Different levels represent the $\chi^{2}$-values of the comparison between the cumulative age distribution in the selected GC sample and a set of 66 simulated systems. For both, the modelling and the determination of the age distribution the SSP models by Maraston (2005) have been applied. Similar to the application of the Bruzual \& Charlot models (2000) the best fitting models contain a large fraction of young/intermediate age globular clusters.

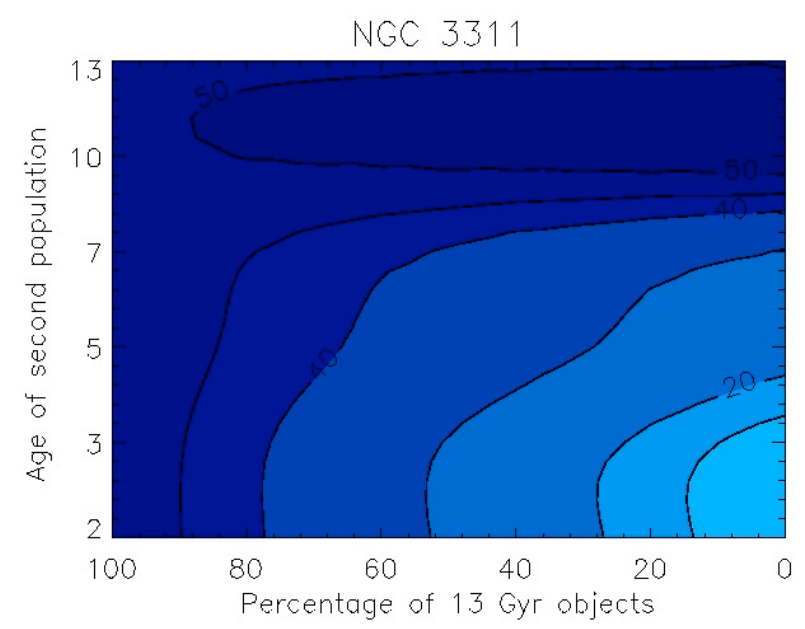

Fig. A.5. Contour plot representing the results of the $\chi^{2}$-test comparing the cumulative age distribution in the NGC 3311 GCS (selected sample) and the Monte-Carlo simulations. As in Fig. A.4 the SSP models by Maraston (2005) have been used.

metallicity (i.e. $[\mathrm{Fe} / \mathrm{H}] \approx-2.3$ ) is much bluer in the Bruzual $\&$ Charlot models. Consequently, the metallicity distribution for the IC 4051 GCs will be shifted toward lower metallicities when the Maraston models are applied. This can be seen in the left panels of Fig. A.6. The metallicity for 2 Gyr old objects (red histogram) peaks at much lower values compared to Fig. 12, left panels. For the first generation of GCs (13 Gyr) the metallicity estimates change only marginally and are also similar for a 5 Gyr population within the anticipated accuracy of 0.2 dex.

Based on the above results we therefore conclude that our primary goal- the detection of age sub-populations in GCSs- is not significantly influenced by the choice of the SSP model. Nevertheless, we note that our method is somewhat 
M. Hempel et al.: Extragalactic globular clusters in the near infrared. V., Online Material p 4
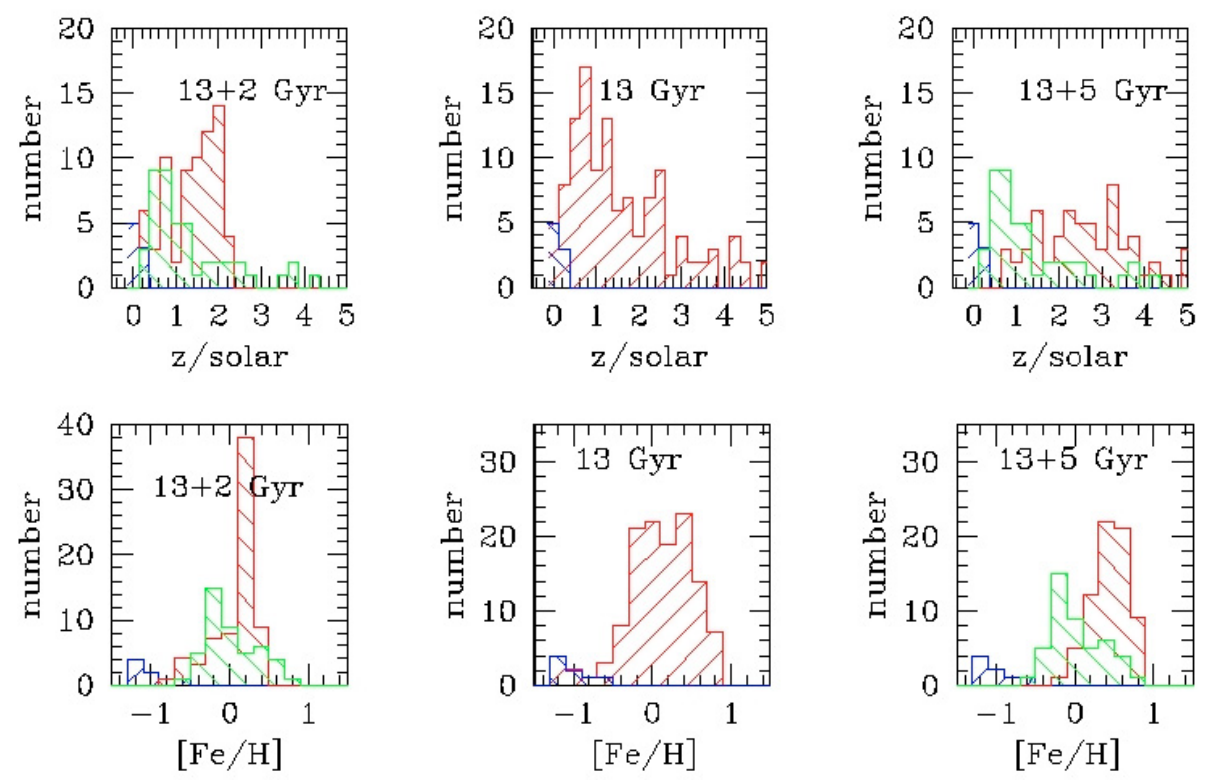

Fig. A.6. Metallicity distribution in IC 4051 assuming different GC age distributions. We show the metallicity distribution both in terms of linear metal abundance (upper panel) as well as in logarithmic metallicity $[\mathrm{Fe} / \mathrm{H}]$ (lower panels). The different panels and the color coding of the histograms correspond to Fig. 12.

handicapped when the SSP model isochrones are not monotonic, e.g. for a given age an unique correlation between the two colors can not be established. There is, however, no doubt that state of the art SSP models should be applied, especially when the information we want to extract from the globular cluster colors become more and more detailed (including metallicity distributions) and an increasing accuracy of the data allows more precise calculations. 